\title{
Maternal and neonatal data collection systems in low- and middle-income countries for maternal vaccines active safety surveillance systems: A scoping review
}

Mabel Berrueta ${ }^{1 *}$ (D), Agustin Ciapponi ${ }^{1}$, Ariel Bardach ${ }^{1}$, Federico Rodriguez Cairoli ${ }^{1}$, Fabricio J. Castellano ${ }^{1}$, Xu Xiong ${ }^{2}$, Andy Stergachis ${ }^{3}$, Sabra Zaraa ${ }^{3}$, Ajoke Sobanjo-ter Meulen ${ }^{4}$, Pierre Buekens ${ }^{2}$ and Scoping Review Collaboration Group

\begin{abstract}
Background: Most post-licensure vaccine pharmacovigilance in low- and middle-income countries (LMICS) are passive reporting systems. These have limited utility for maternal immunization pharmacovigilance in LMIC settings and need to be supplemented with active surveillance. Our study's main objective was to identify existing perinatal data collection systems in LMICs that collect individual information on maternal and neonatal health outcomes and could be developed to inform active safety surveillance of novel vaccines for use during pregnancy.

Methods: A scoping review was performed following the Arksey and O'Malley six-stage approach. We included studies describing electronic or mixed paper-electronic data collection systems in LMICs, including research networks, electronic medical records, and custom software platforms for health information systems. Medline PubMed, EMBASE, Global Health, Cochrane Library, LILACS, Bibliography of Asian Studies (BAS), and CINAHL were searched through August 2019. We also searched grey literature including through Google and websites of existing relevant perinatal data collection systems, as well as contacted authors of key studies and experts in the field to validate the information and identify additional sources of relevant unpublished information.

(Continued on next page)
\end{abstract}

\footnotetext{
* Correspondence: mberrueta@iecs.org.ar

'Instituto de Efectividad Clínica y Sanitaria (IECS), Dr. Emilio Ravignani 2024 (C1014CPV), Buenos Aires, Argentina

Full list of author information is available at the end of the article
}

(c) The Author(s). 2021 Open Access This article is licensed under a Creative Commons Attribution 4.0 International License, which permits use, sharing, adaptation, distribution and reproduction in any medium or format, as long as you give appropriate credit to the original author(s) and the source, provide a link to the Creative Commons licence, and indicate if changes were made. The images or other third party material in this article are included in the article's Creative Commons licence, unless indicated otherwise in a credit line to the material. If material is not included in the article's Creative Commons licence and your intended use is not permitted by statutory regulation or exceeds the permitted use, you will need to obtain permission directly from the copyright holder. To view a copy of this licence, visit http://creativecommons.org/licenses/by/4.0/. The Creative Commons Public Domain Dedication waiver (http://creativecommons.org/publicdomain/zero/1.0/) applies to the data made available in this article, unless otherwise stated in a credit line to the data. 


\begin{abstract}
(Continued from previous page)
Results: A total of 11,817 records were identified. The full texts of 264 records describing 96 data collection systems were assessed for eligibility. Eight perinatal data collection systems met our inclusion criteria: Global Network's Maternal Newborn Health Registry, International Network for the Demographic Evaluation of Populations and their Health; Perinatal Informatic System; Pregnancy Exposure Registry \& Birth Defects Surveillance; SmartCare; Open Medical Record System; Open Smart Register Platform and District Health Information Software 2. These selected systems were qualitatively characterized according to seven different domains: governance; system design; system management; data management; data sources, outcomes and data quality.

Conclusion: This review provides a list of active maternal and neonatal data collection systems in LMICs and their characteristics as well as their outreach, strengths, and limitations. Findings could potentially help further understand where to obtain population-based high-quality information on outcomes to inform the conduct of maternal immunization active vaccine safety surveillance activities and research in LMICs.
\end{abstract}

Keywords: Active surveillance, Electronic Registries, Global Health, Health Information Systems, Pharmacovigilance, Pregnancy, Vaccination

\section{Background}

Spontaneous or passive reporting systems are a cornerstone of vaccine safety surveillance in low- and middleincome countries (LMICs) [1]. This type of reporting relies on health professionals, patients, or others reporting suspected adverse events to public health or governmental organization. These systems have several limitations, including potentially inconsistent diagnostic criteria, underreporting, varying data quality, lack of data to establish a denominator, and little or no background information [1, 2]. In of these limitations, the launch of new vaccines for immunization of pregnant women requires additional safety surveillance efforts to be in place, including active surveillance in pregnant women and newborns and other post-approval safety monitoring mechanisms. Active surveillance aims to detect adverse events on an ongoing basis within a defined group of people. It is especially useful in conjunction with the introduction of new vaccines. Using active surveillance systems, new vaccines in development for maternal immunization (e.g., respiratory syncytial virus (RSV), group B streptococcus (GBS) and severe acute respiratory syndrome coronavirus 2 (SARS-CoV-2) could achieve their main goal of reducing morbidity and mortality in newborns in an informed manner [3]. Traditionally, active surveillance systems in LMIC have been linked to the Expanded Program on Immunization (EPI) and primarily focused on pediatric vaccines administered to children. For the evaluation of vaccine safety in pregnant women, maternal and neonatal data collection systems need to be leveraged to provide knowledge on background rates of pregnancy outcomes and newborn events. In the absence of an accurate background rate of an event, it is impossible to know if the adverse event is occurring at an expected or higher than expected rate. Having an established background rate would be further helpful for informing policies and designing active vaccine safety surveillance studies at sentinel sites $[4,5]$. Perinatal outcomes information is generally unavailable in LMICs, due to many reasons, including the scarcity of resources and trained staff to support robust data collection systems, occurrence of vital and clinical events outside medical facilities, the lack of standardized, comprehensive, national registers and registration systems, inconsistencies among maternal newborn health outcome definitions, and the fact that medical records are often incomplete, poorly maintained and only paper based $[1,2,6]$. Lack of these records makes linking individual data from mother and their babies across systems cumbersome or sometimes impossible.

The Global Alignment of Immunization Safety Assessment in pregnancy (GAIA) project defined case definitions for main $\mathrm{MNCH}$ outcomes [7]. However, no sustainable answers are available on feasibility to implement them in the field $[5,7]$. Various population-based surveys, surveillance systems, health information systems and perinatal data collection systems are already in place and could provide information on maternal and infant health in low-resource settings [8]. Mapping and harmonizing these existing platforms would allow LMICs to increase their ability to monitor relevant $\mathrm{MNCH}$ outcomes following maternal immunization.

There is an urgent need to develop or improve active safety surveillance of novel vaccines in pregnancy by understanding and adapting existing $\mathrm{MNCH}$ data collection systems. As part of a landscape analysis for integrated maternal immunization active safety surveillance and maternal data collection systems in LMICs, a scoping review was conducted to identify existing electronic and mixed paper-electronic data collection systems that register continuous and individual level MNCH data in LMICs with the potential to provide background data on diseases as well as record $\mathrm{MNCH}$ events/outcomes for active safety surveillance for novel maternal vaccines. 


\section{Methods}

We included studies describing electronic or mixed paper-electronic data collection systems in LMICs, including research networks, electronic medical records, and custom software platforms for health information systems. Medline PubMed, EMBASE, Global Health, Cochrane Library, LILACS, Bibliography of Asian Studies (BAS), and CINAHL were searched through August 2019. We also searched grey literature including through Google and websites of existing relevant perinatal data collection systems, as well as contacted authors of key studies and experts in the field to validate information and identify additional sources of relevant unpublished information.

We performed a scoping review following the Arksey and O'Malley six-stage approach [9] and the Preferred Reporting Items for Systematic Reviews and MetaAnalyses (PRISMA) statement guidelines and its extension for scoping reviews [10] (Additional file 1). The scoping review protocol was previously published in the Gates Open Research Journal [11].

Two main research questions guided the scoping review:

- What existing prenatal and postnatal data collection systems are in place at the facility level and community level that could provide continuous, longitudinal, and individual information on maternal and neonatal health outcomes in LMICs?

- Do existing prenatal and postnatal data collection systems have the capacity to inform active safety surveillance for maternal vaccines and other maternal health interventions?

Studies describing electronic or mixed paperelectronic perinatal data collection systems in LMICs, including research networks, electronic medical records, and custom software platforms for health information systems were included. Search strategies were run in databases (Medline, PubMed, EMBASE, Global Health, Cochrane Library, LILACS, Bibliography of Asian Studies (BAS), and CINAHL) and Google through August 2019. Grey literature including websites of existing data collection systems were explored [11].

The PRISMA Extension for Scoping Reviews (PRIS MA-ScR) flow diagram represents the formal literature review and screening process developed (Fig. 1). From those perinatal data collection systems identified in the full text article review, all specific data points published in the protocol [11] and listed in Table 1 were recorded.

A modified framework to assess and describe the final identified existing $\mathrm{MNCH}$ eligible health systems attributes was used based on frameworks available in the literature [12-14]. Governance; System design; Data management; Data sources, Outcomes and Data quality are the six domains used to present the extracted data points (Table 2).

The objectives of the consultation phase were to share preliminary findings and comparative analysis of final list of systems with experts in order to validate the domains used to describe the systems (Table 2) and the extracted data points (Table 1) from each system, as well as to identify additional grey sources of information.

\section{Results}

\section{Study selection and characteristics}

A total of 11,817 records including additional sources from reference lists and grey literature were identified. After removing duplicates, the 8,069 records left were screened by title and abstract, and 7,805 were considered irrelevant mainly because they were not related to $\mathrm{MNCH}$ data collection systems in LMICs. The full texts of 264 records describing 96 data collection systems were assessed for eligibility and finally, eight perinatal data collection systems (involving 165 reports) were included in qualitative synthesis (Figure 1). The included 165 reports were categorized as descriptive articles (51 published and 12 unpublished), published research studies related to the data collection systems $(n=87)$, official system websites $(n=7)$, user manuals or guides $(n=3)$, and other web links that were not official systems websites $(n=5)$. The most frequent reasons for excluding 99 reports were not collecting perinatal outcomes continuously at the individual-level (64), not currently capturing data (12) or not being a specific $\mathrm{MNCH}$ data collection system (9). The reasons for final exclusions of the potentially eligible systems are presented in Additional file 3.

The eight data collection systems finally selected were: 1) Global Network's Maternal Newborn Health Registry (GN-MNHR), 2) International Network for the Demographic Evaluation of Populations and their Health (INDEPTH), 3) Perinatal Informatic System (SIP), 4) Pregnancy Exposure Registry \& Birth Defects Surveillance (PER/BDS), 5) SmartCare, 6) Open Medical Record System (OpenMRS), 7) Open Smart Register Platform (OpenSRP) and 8) District Health Information Software 2 (DHIS 2) (see Table 3).

Regarding the geographic distribution of the systems, although they were implemented in many different countries and districts, not all sites captured individual maternal and neonatal data. Therefore, we only included the sites that met the objectives and inclusion criteria of our study. DHIS 2 tracker, GN-MHNR and INDEPTH are in sub-Sahara Africa and South Asia. Additionally, GN-MNHR is also located in Latin America and the Caribbean (Guatemala), DHIS 2 is in the Middle East and North Africa (West Bank and Gaza), and INDEPTH 


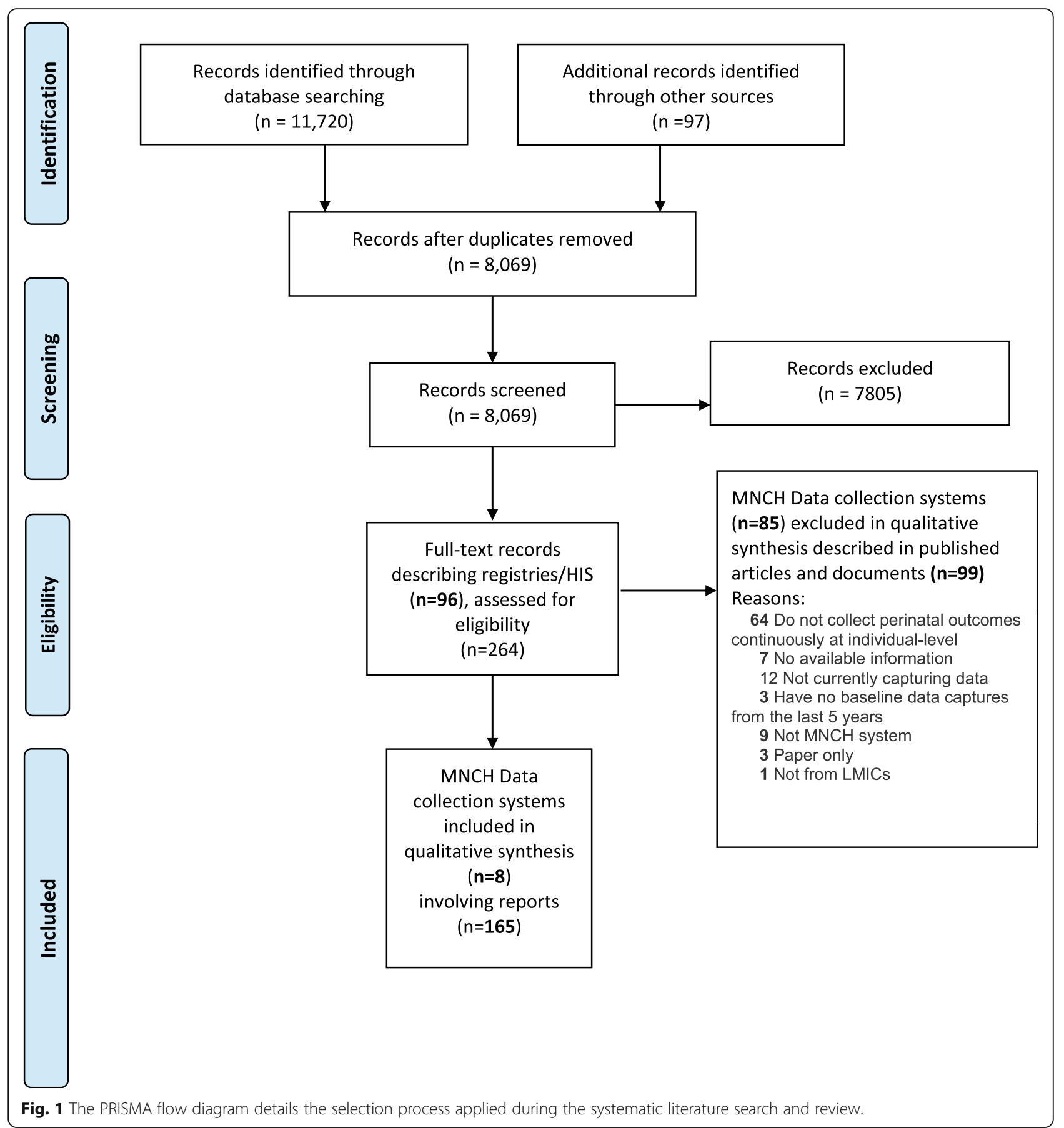

is in East Asia and the Pacific (Indonesia, Malaysia and Vietnam). PER/BDS is located only in South Africa. SmartCare is in Zambia and OpenSRP is in Indonesia and Pakistan. Finally, OpenMRS is located in Uganda, Rwanda, Lesotho, Malawi, Kenya and Haiti.

Out of the 165 included reports: 86 (52.1\%) were related to INDEPTH [16-101], 26 (15.7\%) to Global Network [102-127], $24(14.5 \%)$ to DHIS 2 [128-151], 9 (5.4\%) to OpenMRS [152-160], $6(3.6 \%)$ to SIP [161-
166], 6 (3.6\%) to OpenSRP [167-172], 4 (2.4\%) to PER/ BDS [173-176], and $4(2.4 \%)$ to SmartCare [177-180].

\section{Major findings by identified domain}

Following the analysis of the extracted data, the eight included systems' synthetized results were presented in seven domains: Governance; System design; System management; Data management; Data sources, Outcomes and Data quality (Tables 4, 5, 6, 7, 8 and 9). Extracted 
Table 1 Data points abstracted from selected studies [11].
a) Countries where the systems operate
b) The extent of data collection (e.g. community services, facilities services)
c) The main purpose of the system d) Records linkage
e) The level of implementation (e.g. local, national)
f) Target population (e.g. specified subgroups or total population)
g) The data collection form used (e.g. electronic, paper)
h) Whether the data entered into the registry was primary data, or if the registry was based on secondary data from pre-existing sources.
i) MNCHcare periods that the system registers
j) Whether it collects the MNCH variables defined by the GAIA project
k) Whether it collects socio-demographic data, obstetric information, pre-existing conditions of each women, and antenatal care outcome process.
I) Type of code for classification of diseases used
m) The individual data capture process (how and where it is done)
n) Baseline data (pregnancy and outcomes) timeframe
o) Capability to import, compile and export electronic data.
p) Capability to link individual MNCH data with other registers(e.g. laboratory records, expense drug records, vaccine registers)
q) Type of monitoring to assess data quality
r) Whether it has a back-up policy.
s) Availability of data collection tools and system documentation
t) Maintenance and update process
u) Whether it has data access policies,
v) Type of privacy protection
w) Funding or sponsor of each system
x) Prior use in active surveillance or pharmacovigilance or post- marketing surveillance.

data has been made available in a web-interactive App: http://safeinpregnancy.org/la_sc/table_by_domain.html\#.

\section{Governance (Table 4)}

These systems are supported by different categories of institutions [181] such as private foundations (e.g., Bill and Melinda Gates Foundation, Wellcome Trust, The Rockefeller Foundation, Children's Investment Fund Foundation, Hewlett Foundation), governmental agencies in high-income countries (e.g., United States Agency for International Development (USAID), Centers for Disease Control and Prevention, Norad), global health initiatives (President's Emergency Plan For AIDS Relief, The Global Alliance for Vaccines and Immunization), research councils (National Institutes of Health, National Institute of Child Health and Human Development, Medical Research Council), non-governmental organizations (Comic Relief), international organizations (World Health Organization /Pan America Health Organization, UNICEF), universities (Harvard University, University of Oslo), private sector organizations (GlaxoSmithKline, Qualcomm) and LMIC governments (South Africa National Department of Health) $[14,17,37,77,103,161$, 178].

Some organizations were also responsible for the development and implementation of the included systems and are responsible for its optimal operability, such as the University of Oslo in the case of DHIS 2 [131], Partners in Health for OpenMRS [152], WHO for OpenSRP [170] and National Department of Health South Africa for PER/BDS [182] (Table 4). The majority of the systems demonstrated features that support the protection and privacy of collected information through

Table 2 Framework: domains used to present the extracted data points

\section{Domains}

Governance

System design Infrastructure development and maintenance; multilanguage; capacity to compile and transfer electronic data; data access;

Partnerships, both private and across government agencies, and coalitions; system policy and digital standards, including privacy and security; information and communication technology standards and documentation; geographical area of influence; primary purpose of the system (clinical care, research, information systems, mortality registries, insurance registries, birth registration) records linkage and interoperability integration; type of platform to be used; what other systems may link to, and how the systems integrate or interoperate.

Data

management

Data sources

Outcomes Maternal and perinatal outcomes based on GAIA project [15]

Data collection method used (paper, electronic, both, or not defined); Data entry: based on primary data entered into the registry or based on a secondary/duplicate data collection from existing source, standardized classification; who capture individual data (physician, patient)

The scale of the implementation of the registry (national, district, local); health sector involved (private, public, social security), the specified population captured by the registry data collection (total population, only subgroups/select population); variables that collect; prior use in post marketing surveillance. Neonatal death; Congenital anomalies; Neonatal infections; Preterm birth; Stillbirth; Low birth weight; Small for gestational age; Respiratory distress; Failure to thrive; Microencephaly; Neonatal encephalopathy; Neonatal seizures; Neurodevelopmental delay. Maternal death; Fetal distress; Postpartum hemorrhage; Antenatal bleeding; Dysfunctional labour; Spontaneous abortion; Preeclamsia/Eclampsia; Foetal growth retardation; Gestational diabetes; Postpartum endometritis; Gestational Hypertension; Chorioamnionitis; Ectopic pregnancy; Premature preterm rupture of membranes; Preterm labor; Insufficient cervix 
Table 3 Included data collection systems

\begin{tabular}{|c|c|c|c|c|}
\hline & $\begin{array}{l}\text { Data collection } \\
\text { system }\end{array}$ & Description & $\begin{array}{l}\text { Countries where serves to } \\
\text { capture MNCH individual data }\end{array}$ & $\begin{array}{l}\text { Number of pregnant women/ } \\
\text { deliveries registered }\end{array}$ \\
\hline \multirow[t]{2}{*}{$\begin{array}{l}\text { Research } \\
\text { network }\end{array}$} & $\begin{array}{l}\text { Global Network's } \\
\text { Maternal Newborn } \\
\text { Health Registry }\end{array}$ & $\begin{array}{l}\text { The Global Network's Maternal } \\
\text { Newborn Health Registry (MNHR) is } \\
\text { a prospective, population-based regis- } \\
\text { try of pregnancies at the Global } \\
\text { Network sites. It started in 2008, } \\
\text { enrolls and follows up on pregnant } \\
\text { women and their newborns up to } \\
42 \text { days postpartum. }\end{array}$ & $\begin{array}{l}\text { It captures data in six countries, } \\
\text { including sites in Pakistan; Kenya; } \\
\text { Zambia ; Democratic Republic of } \\
\text { the Congo; Guatemala; and two in } \\
\text { India. }\end{array}$ & $\begin{array}{l}370,000 \text { mother and their infants } \\
\text { have been enrolled so far }\end{array}$ \\
\hline & $\begin{array}{l}\text { International } \\
\text { Network for the } \\
\text { Demographic } \\
\text { Evaluation of } \\
\text { Populations and } \\
\text { their Health }\end{array}$ & $\begin{array}{l}\text { INDEPTH is a network of } \\
\text { independent Health and } \\
\text { Demographic Surveillance System } \\
\text { (HDSS) sites that carry out } \\
\text { longitudinal research. The INDEPTH } \\
\text { Network Maternal, Newborn \& Child } \\
\text { Health Working Group (MNCH-WG) } \\
\text { coordinate the surveillance of } \\
\text { pregnancies and outcome tracking. }\end{array}$ & $\begin{array}{l}\text { Bangladesh; Burkina Faso; Cote } \\
\text { d'Ivoire; Ethiopia; Gambia; Ghana; } \\
\text { Guinea-Bissau; India; Indonesia; } \\
\text { Kenya; Malawi; Malaysia; } \\
\text { Mozambique; Nigeria; Senegal; } \\
\text { South Africa; Tanzania; Uganda; } \\
\text { Vietnam }\end{array}$ & $\begin{array}{l}\text { 97,499 Estimated number of total } \\
\text { births over five years (2012-2017) } \\
\text { captured within the HDSS (only in } \\
5 \text { sites: Bandim; Matlab; Kintampo; } \\
\text { Dabat; Igangamayuge) }\end{array}$ \\
\hline \multirow[t]{3}{*}{$\begin{array}{l}\text { Electronical } \\
\text { medical } \\
\text { record }\end{array}$} & $\begin{array}{l}\text { Perinatal Informatic } \\
\text { System }\end{array}$ & $\begin{array}{l}\text { Perinatal Informatic System ("SIP", by } \\
\text { the acronym in Spanish: Sistema } \\
\text { Informático Perinatal) by Pan } \\
\text { American Health Organization } \\
\text { (PAHO) is a perinatal clinical record } \\
\text { that Ministries of health and } \\
\text { maternity services (public and } \\
\text { private) have adopted. }\end{array}$ & $\begin{array}{l}\text { Cuba; El Salvador; Guatemala; } \\
\text { Honduras; Nicaragua; Panama; } \\
\text { Dominician Republic; Mexico; } \\
\text { Argentina; Bolivia; Brazil; Chile; } \\
\text { Colombia; Ecuador; Paraguay; Peru; } \\
\text { Uruguay; Venezuela }\end{array}$ & Not Available \\
\hline & $\begin{array}{l}\text { Pregnancy Exposure } \\
\text { Registry \& Birth } \\
\text { Defects Surveillance }\end{array}$ & $\begin{array}{l}\text { PER/BDS is an electronic medical } \\
\text { record system designed to collect } \\
\text { data on pregnancy exposures during } \\
\text { routine care. }\end{array}$ & South Africa & $\begin{array}{l}42,300 \text { estimated deliveries per } \\
\text { year in Gauteng and in Western } \\
\text { Cape }\end{array}$ \\
\hline & SmartCare & $\begin{array}{l}\text { SmartCare is a portable, integrated } \\
\text { and electronical medical system } \\
\text { developed by Zambia's Ministry of } \\
\text { Health }\end{array}$ & Zambia & Not Available \\
\hline \multirow[t]{3}{*}{$\begin{array}{l}\text { Custom } \\
\text { Software } \\
\text { platform }\end{array}$} & $\begin{array}{l}\text { District Health } \\
\text { Information Software } \\
2 \text { tracker }\end{array}$ & $\begin{array}{l}\text { DHIS2 is a customize platform } \\
\text { typically used as national health } \\
\text { information system. Tracker module } \\
\text { allows to collect pregnant and } \\
\text { babies data and track longitudinally } \\
\text { the progress of a patients over time. }\end{array}$ & $\begin{array}{l}\text { Bangladesh; Liberia; West Bank and } \\
\text { Gaza; South Africa }\end{array}$ & Not Available \\
\hline & $\begin{array}{l}\text { Open Medical } \\
\text { Record System }\end{array}$ & $\begin{array}{l}\text { OpenMRS is a software platform and } \\
\text { a reference application which } \\
\text { enables the design of a customized } \\
\text { medical records system with no } \\
\text { programming knowledge. }\end{array}$ & $\begin{array}{l}\text { Uganda; Malawi; Rwanda; Lesotho; } \\
\text { Kenya; Haiti }\end{array}$ & Not Available \\
\hline & $\begin{array}{l}\text { Open Smart Register } \\
\text { Platform }\end{array}$ & $\begin{array}{l}\text { It is a mobile-first platform, built to } \\
\text { enable data-driven decision making } \\
\text { at all levels of the health system } \\
\text { with the following health modules: } \\
\text { Reproductive, maternal, neonatal, } \\
\text { and child health, childhood immuni- } \\
\text { zations, tuberculosis, nutrition, mal- } \\
\text { aria and early childhood } \\
\text { development. }\end{array}$ & Indonesia; Pakistán & Not Available \\
\hline
\end{tabular}

anonymization of data, implementation of passwords before access, or external security (cybersecurity) [38, 77, $103,131,141,154,170,177,179]$. Up to the search date, neither GN-MNHR nor SIP allowed for data encryption.

We were able to access the operating manuals, data forms, and documentation for six out of the eight systems [17, 103, 131, 152, 163, 170]; for SmartCare and
PER/BDS these types of documents were not identified. Although most of the systems were designed for clinical care, some had been conceptualized for research such as GN-MNHR [106, 113, 119, 122, 123] or surveillance such as INDEPTH [46, 49, 77, 100] or PER/BDS [174]. Some of these were designed to satisfy more than one objective, and in the case of OpenMRS, this varied in 
Table 4 Governance

\begin{tabular}{|c|c|c|c|c|c|c|c|c|}
\hline Variable & 1: DHIS2 & $\begin{array}{l}\text { 2: GN } \\
\text { MNHR }\end{array}$ & 3: INDEPTH & 4: OpenMRS & 5: OpenSRP & 6: SIP & 7: PER/BDS & 8: SmartCare \\
\hline $\begin{array}{l}\text { Sponsor/ } \\
\text { funding }\end{array}$ & $\begin{array}{l}\text { Norad, PEPF } \\
\text { AR, BMGF, } \\
\text { CDC, GAVI, } \\
\text { UNICEF, } \\
\text { WHO, UiO }\end{array}$ & $\begin{array}{l}\text { NICHD, } \\
\text { BMGF }\end{array}$ & $\begin{array}{l}\text { WHO, BMGF, NIH, } \\
\text { Wellcome Trust, Harvard } \\
\text { University, Sida, GSK, } \\
\text { Children's investment } \\
\text { fund foundation, Comic } \\
\text { relief, Hewlett Foundation }\end{array}$ & $\begin{array}{l}\text { CDC, USAID, } \\
\text { The Rockefeller } \\
\text { Foundation }\end{array}$ & $\begin{array}{l}\text { Wellcome } \\
\text { Trust, } \\
\text { UNICEF, } \\
\text { WHO, UBS, } \\
\text { Qualcomm, } \\
\text { PATH }\end{array}$ & $\begin{array}{l}\text { CLAP/ } \\
\text { PAHO }\end{array}$ & $\begin{array}{l}\text { PEPFAR, } \\
\text { WHO, South } \\
\text { Africa } \\
\text { National } \\
\text { Department } \\
\text { of Health }\end{array}$ & CDC \\
\hline $\begin{array}{l}\text { Institution(s) } \\
\text { in charge of } \\
\text { system } \\
\text { development } \\
\text { and updates }\end{array}$ & $\begin{array}{l}\text { University of } \\
\text { Oslo }\end{array}$ & $\begin{array}{l}\text { RTI } \\
\text { International }\end{array}$ & INDEPTH Network & $\begin{array}{l}\text { Partners In } \\
\text { Health, } \\
\text { Regenstrief } \\
\text { Institute, South } \\
\text { African } \\
\text { Medical } \\
\text { Reserch } \\
\text { Council }\end{array}$ & $\begin{array}{l}\text { Interactive } \\
\text { Health } \\
\text { Solutions, } \\
\text { WHO }\end{array}$ & $\begin{array}{l}\text { CLAP/ } \\
\text { PAHO }\end{array}$ & $\begin{array}{l}\text { National } \\
\text { Department } \\
\text { of Health } \\
\text { South Africa }\end{array}$ & $\begin{array}{l}\text { BroadReach } \\
\text { Consulting } \\
\text { LLC }\end{array}$ \\
\hline $\begin{array}{l}\text { Data privacy } \\
\text { protection }\end{array}$ & Yes & Yes & Yes & Yes & Yes & No & Yes & Yes \\
\hline $\begin{array}{l}\text { Description of } \\
\text { the } \\
\text { mechanism/ } \\
\text { Process }\end{array}$ & $\begin{array}{l}\text { Anonymized } \\
\text { data }\end{array}$ & $\begin{array}{l}\text { Anonymized } \\
\text { data }\end{array}$ & Anonymized data & $\begin{array}{l}\text { Anonymized } \\
\text { data }\end{array}$ & $\begin{array}{l}\text { Anonymized } \\
\text { data }\end{array}$ & $\begin{array}{l}\text { Not } \\
\text { applicable }\end{array}$ & $\begin{array}{l}\text { Anonymized } \\
\text { data }\end{array}$ & $\begin{array}{l}\text { Anonymized } \\
\text { data }\end{array}$ \\
\hline $\begin{array}{l}\text { External } \\
\text { Protection } \\
\text { (Cybersecurity) }\end{array}$ & Yes & Yes & Yes & N/A & Yes & No & Yes & Yes \\
\hline Access Policies & Yes & Yes & Yes & Yes & Yes & No & Yes & Yes \\
\hline $\begin{array}{l}\text { Description of } \\
\text { the } \\
\text { mechanism/ } \\
\text { process }\end{array}$ & $\begin{array}{l}\text { User/ } \\
\text { password }\end{array}$ & $\begin{array}{l}\text { User/ } \\
\text { password }\end{array}$ & User/password & User/password & $\begin{array}{l}\text { User/ } \\
\text { password }\end{array}$ & $\begin{array}{l}\text { Not } \\
\text { applicable }\end{array}$ & $\begin{array}{l}\text { User/ } \\
\text { password }\end{array}$ & $\begin{array}{l}\text { User/ } \\
\text { password }\end{array}$ \\
\hline $\begin{array}{l}\text { Backup } \\
\text { policies }\end{array}$ & Yes & Yes & Yes & Yes & Yes & Yes & Yes & Yes \\
\hline $\begin{array}{l}\text { Primary } \\
\text { purpose of the } \\
\text { system/data } \\
\text { collection }\end{array}$ & Clinical care & Research & Surveillance & Clinical care & Clinical care & $\begin{array}{l}\text { Clinical } \\
\text { care }\end{array}$ & Surveillance & Clinical care \\
\hline
\end{tabular}

different locations where the system is in place [130, $145,152,161,170,178]$.

\section{System design (Table 5)}

The type of license was free and open-source for four systems: DHIS 2 [131, 140], OpenSRP [170], OpenMRS [156] and PER/BDS [182]. SIP used a closed-code source [161] and GN-MNHR used a private license. Web-based platforms were the most frequently used $[46,49,131$, $140,153,156,170]$. However, the two systems GNMNHR [103] and SIP [161, 163] still used local networks. No information on the type of license was recorded on SmartCare and INDEPTH.

Interoperability was assessed through the system's ability to compile, transfer and export data, and integrate with other data sources, systems, individual and laboratory records, and/or national health record databases. The DHIS 2 [131, 132, 145, 146, 148], INDEPTH [17, 18, 21, 28, 35-38, 46, 58, 97, 100], SmartCare [177-180], OpenMRS [153, 154], OpenSRP [171] and PER/BDS [174] all have these capabilities. GN-MNHR [103, 108] and SIP [161, 163] systems showed lack of ability to link with National Health databases and clinical or laboratory records. All eight data collection systems demonstrated flexibility to add new variables.

Data were captured only at facilities in the system SIP [161], SmartCare [178] and PER/BDS [174, 182]. Data were captured both at the facility and community level for the systems DHIS $2[144,148]$, INDEPTH $[49,100]$, OpenMRS [152-154] and OpenSRP [170]. GD-MNHR only captured data at the community level $[108,113]$. GD-MNHR only captured data at the community level $[17,33,109,123,130,131]$.

\section{Data management (Table 6)}

Although all of the included systems recorded data electronically $[28,49,62,85,100,108,141,145,150,152$, 161, 169] and SmartCare [178, 179], used a mixed modality and initially captured data only on paper. Trained health providers, including nurses and doctors, collected the data in all systems. Only the DHIS 2 system through the MomConnect platform [140] allows pregnant 
Table 5 System design

\begin{tabular}{|c|c|c|c|c|c|c|c|c|}
\hline Variable & 1: DHIS2 & 2: GN MNHR & 3: INDEPTH & 4: OpenMRS & 5: OpenSRP & 6: SIP & $\begin{array}{l}\text { 7: PER/ } \\
\text { BDS }\end{array}$ & $\begin{array}{l}\text { 8: } \\
\text { SmartCare }\end{array}$ \\
\hline Type of platform & $\begin{array}{l}\text { Web } \\
\text { based }\end{array}$ & $\begin{array}{l}\text { Local } \\
\text { installation }\end{array}$ & Web based & Web based & Web based & $\begin{array}{l}\text { Local } \\
\text { installation }\end{array}$ & $\begin{array}{l}\text { Web } \\
\text { based }\end{array}$ & N/A \\
\hline Type of license & $\begin{array}{l}\text { Free/ } \\
\text { Open } \\
\text { Source }\end{array}$ & Private & N/A & $\begin{array}{l}\text { Free/Open } \\
\text { Source }\end{array}$ & $\begin{array}{l}\text { Free/Open } \\
\text { Source }\end{array}$ & $\begin{array}{l}\text { Free/Close } \\
\text { Code }\end{array}$ & $\begin{array}{l}\text { Free/ } \\
\text { Open } \\
\text { Source }\end{array}$ & N/A \\
\hline Type of database & $\begin{array}{l}\text { Postgre } \\
\text { SQL }\end{array}$ & $\begin{array}{l}\text { SQL Server, } \\
\text { Other }\end{array}$ & $\begin{array}{l}\text { My SQL, SQL } \\
\text { Server, Postgre } \\
\text { SQL, Other }\end{array}$ & $\begin{array}{l}\text { My SQL, SQL } \\
\text { Server }\end{array}$ & $\begin{array}{l}\text { My SQL, } \\
\text { Other }\end{array}$ & Other & N/A & SQL Server \\
\hline $\begin{array}{l}\text { Operating system in which } \\
\text { it runs }\end{array}$ & $\begin{array}{l}\text { Windows, } \\
\text { Android }\end{array}$ & Windows & Linux, Android & $\begin{array}{l}\text { Windows, } \\
\text { Linux }\end{array}$ & Android & Windows & N/A & Windows \\
\hline Data Encryption & Yes & No & Yes & Yes & Yes & No & Yes & N/A \\
\hline Language & $\begin{array}{l}\text { English, } \\
\text { Other }\end{array}$ & $\begin{array}{l}\text { English, } \\
\text { French, } \\
\text { Spanish, } \\
\text { Other }\end{array}$ & English, Other & $\begin{array}{l}\text { English, } \\
\text { Other }\end{array}$ & $\begin{array}{l}\text { English, } \\
\text { French, } \\
\text { Other }\end{array}$ & $\begin{array}{l}\text { English, } \\
\text { French, } \\
\text { Spanish, } \\
\text { Other }\end{array}$ & English & English \\
\hline Data Capture Site & $\begin{array}{l}\text { Hospitals } \\
\text { Clinics }\end{array}$ & $\begin{array}{l}\text { Hospitals } \\
\text { Clinics } \\
\text { Community }\end{array}$ & Community & $\begin{array}{l}\text { Hospitals } \\
\text { Clinics } \\
\text { Community }\end{array}$ & $\begin{array}{l}\text { Hospitals } \\
\text { Clinics } \\
\text { Community }\end{array}$ & $\begin{array}{l}\text { Hospitals } \\
\text { Clinics }\end{array}$ & $\begin{array}{l}\text { Hospitals } \\
\text { Clinics }\end{array}$ & $\begin{array}{l}\text { Hospitals } \\
\text { Clinics }\end{array}$ \\
\hline Allow to export data & Yes & Yes & Yes & Yes & Yes & Yes & Yes & Yes \\
\hline $\begin{array}{l}\text { Records Linkage (Individual } \\
\text { identifiers) }\end{array}$ & Yes & Yes & Yes & Yes & Yes & Yes & Yes & Yes \\
\hline $\begin{array}{l}\text { Ability to integrate with } \\
\text { other data sources }\end{array}$ & Yes/API & Yes & Yes & Yes/API & Yes & No & Yes & Yes \\
\hline $\begin{array}{l}\text { Ability to integrate with } \\
\text { National Health System } \\
\text { databases }\end{array}$ & Yes & No & Yes & Yes & Yes & No & Yes & Yes \\
\hline $\begin{array}{l}\text { Ability to link with } \\
\text { laboratory registries }\end{array}$ & Yes & No & Yes & Yes & Yes & No & Yes & Yes \\
\hline $\begin{array}{l}\text { Capacity to compile and } \\
\text { transfer data }\end{array}$ & Yes & Yes & Yes & Yes & Yes & Yes & Yes & Yes \\
\hline
\end{tabular}

$\mathrm{N} / \mathrm{A}$ : Not available information

Table 6 Data management

\begin{tabular}{|c|c|c|c|c|c|c|c|c|}
\hline Variable & 1: DHIS2 & $\begin{array}{l}\text { 2: GN } \\
\text { MNHR }\end{array}$ & 3: INDEPTH & $\begin{array}{l}\text { 4: } \\
\text { OpenMRS }\end{array}$ & $\begin{array}{l}\text { 5: } \\
\text { OpenSRP }\end{array}$ & 6: SIP & $\begin{array}{l}\text { 7: PER/ } \\
\text { BDS }\end{array}$ & $\begin{array}{l}\text { 8: } \\
\text { SmartCare }\end{array}$ \\
\hline Data Capture format & $\begin{array}{l}\text { Paper, } \\
\text { Electronic }\end{array}$ & $\begin{array}{l}\text { Paper, } \\
\text { Electronic }\end{array}$ & Paper, Electronic & $\begin{array}{l}\text { Paper, } \\
\text { Electronic }\end{array}$ & Electronic & $\begin{array}{l}\text { Paper, } \\
\text { Electronic }\end{array}$ & Paper & Paper \\
\hline $\begin{array}{l}\text { Standarized } \\
\text { Classifications }\end{array}$ & $\mathrm{ICD} 10$ & No & $\mathrm{ICD} 10$ & $\begin{array}{l}\text { ICD10, } \\
\text { SNOMED- } \\
\text { ST, Other }\end{array}$ & N/A & ICD10 & $\mathrm{ICD} 10$ & N/A \\
\hline $\begin{array}{l}\text { Who capture } \\
\text { individual data }\end{array}$ & $\begin{array}{l}\text { Physician, } \\
\text { Health } \\
\text { provider, } \\
\text { Patient }\end{array}$ & $\begin{array}{l}\text { Physician, } \\
\text { Health } \\
\text { provider }\end{array}$ & Physician, Health provider & $\begin{array}{l}\text { Health } \\
\text { provider }\end{array}$ & $\begin{array}{l}\text { Health } \\
\text { provider, } \\
\text { Patient }\end{array}$ & $\begin{array}{l}\text { Physician, } \\
\text { Health } \\
\text { provider }\end{array}$ & $\begin{array}{l}\text { Health } \\
\text { provider }\end{array}$ & $\begin{array}{l}\text { Physician, } \\
\text { Health } \\
\text { provider }\end{array}$ \\
\hline $\begin{array}{l}\text { Has been used } \\
\text { previously for any } \\
\text { post marketing } \\
\text { surveillance? }\end{array}$ & N/A & No & Yes & N/A & N/A & N/A & No & N/A \\
\hline If yes, for what? & $\begin{array}{l}\text { Not } \\
\text { applicable }\end{array}$ & $\begin{array}{l}\text { Not } \\
\text { applicable }\end{array}$ & $\begin{array}{l}\text { Malaria treatments, Seasonal trivalent } \\
\text { influenza vaccine, Heamophilus } \\
\text { influenza type b vaccine, PREVENT } \\
\text { proyect, other }\end{array}$ & $\begin{array}{l}\text { Not } \\
\text { applicable }\end{array}$ & $\begin{array}{l}\text { Not } \\
\text { applicable }\end{array}$ & $\begin{array}{l}\text { Not } \\
\text { applicable }\end{array}$ & $\begin{array}{l}\text { Not } \\
\text { applicable }\end{array}$ & $\begin{array}{l}\text { Not } \\
\text { applicable }\end{array}$ \\
\hline $\begin{array}{l}\text { If yes what type of } \\
\text { post marketing } \\
\text { surveillance? }\end{array}$ & $\begin{array}{l}\text { Not } \\
\text { applicable }\end{array}$ & $\begin{array}{l}\text { Not } \\
\text { applicable }\end{array}$ & Active surveillance & $\begin{array}{l}\text { Not } \\
\text { applicable }\end{array}$ & $\begin{array}{l}\text { Not } \\
\text { applicable }\end{array}$ & $\begin{array}{l}\text { Not } \\
\text { applicable }\end{array}$ & $\begin{array}{l}\text { Not } \\
\text { applicable }\end{array}$ & $\begin{array}{l}\text { Not } \\
\text { applicable }\end{array}$ \\
\hline
\end{tabular}


Table 7 Data sources

\begin{tabular}{|c|c|c|c|c|c|c|c|c|}
\hline Variable & 1: DHIS2 & $\begin{array}{l}\text { 2: GN } \\
\text { MNHR }\end{array}$ & 3: INDEPTH & 4: OpenMRS & 5: OpenSRP & 6: SIP & 7: PER/BDS & 8: SmartCare \\
\hline $\begin{array}{l}\text { The scale of the } \\
\text { implementation of } \\
\text { the registry }\end{array}$ & $\begin{array}{l}\text { Local, } \\
\text { District, } \\
\text { National, } \\
\text { International }\end{array}$ & $\begin{array}{l}\text { Local, } \\
\text { District, } \\
\text { National, } \\
\text { International }\end{array}$ & $\begin{array}{l}\text { Local, District, } \\
\text { National, } \\
\text { International }\end{array}$ & $\begin{array}{l}\text { Local, District, } \\
\text { National }\end{array}$ & $\begin{array}{l}\text { District, } \\
\text { National }\end{array}$ & $\begin{array}{l}\text { Local, } \\
\text { District, } \\
\text { National, } \\
\text { International }\end{array}$ & District & National \\
\hline $\begin{array}{l}\text { Health sector } \\
\text { involved }\end{array}$ & $\begin{array}{l}\text { Public, } \\
\text { Private }\end{array}$ & $\begin{array}{l}\text { Public, } \\
\text { Private }\end{array}$ & Public, Private & Public & Public & Public & Public & Public \\
\hline $\begin{array}{l}\text { Maternal } \\
\text { sociodemographic } \\
\text { variables } \\
\text { included? }\end{array}$ & $\begin{array}{l}\text { Age, } \\
\text { Education, } \\
\text { Place of } \\
\text { residence }\end{array}$ & $\begin{array}{l}\text { Age, } \\
\text { Education, } \\
\text { Place of } \\
\text { residence }\end{array}$ & $\begin{array}{l}\text { Age, } \\
\text { Education, } \\
\text { Place of } \\
\text { residence }\end{array}$ & $\begin{array}{l}\text { Age, Place of } \\
\text { residence }\end{array}$ & $\begin{array}{l}\text { Age, } \\
\text { Education, } \\
\text { Place of } \\
\text { residence }\end{array}$ & $\begin{array}{l}\text { Age, } \\
\text { Education, } \\
\text { Place of } \\
\text { residence }\end{array}$ & $\begin{array}{l}\text { Age, Education, } \\
\text { Place of residence }\end{array}$ & Age \\
\hline $\begin{array}{l}\text { Data capture } \\
\text { antenatal care } \\
\text { visits }\end{array}$ & Yes & Yes & Yes & Yes & Yes & Yes & Yes & Yes \\
\hline $\begin{array}{l}\text { Timming for } \\
\text { antenatal data } \\
\text { collection }\end{array}$ & At each visit & Retrospective & Retrospective & $\begin{array}{l}\text { Retrospective, } \\
\text { At each visit }\end{array}$ & At each visit & At each visit & At each visit & At each visit \\
\hline $\begin{array}{l}\text { Antenatal care } \\
\text { testing captured? }\end{array}$ & $\begin{array}{l}\text { Shypilis, HIV, } \\
\text { Proteinuiria, } \\
\text { Anemia }\end{array}$ & $\begin{array}{l}\text { Shypilis, HIV, } \\
\text { Anemia }\end{array}$ & $\begin{array}{l}\text { Shypilis, HIV, } \\
\text { Anemia }\end{array}$ & $\begin{array}{l}\text { Shypilis, HIV, } \\
\text { Proteinuiria, } \\
\text { Anemia }\end{array}$ & Shypilis, HIV & $\begin{array}{l}\text { Shypilis, HIV, } \\
\text { Anemia }\end{array}$ & HIV & HIV \\
\hline Birth information & Yes & Yes & Yes & Yes & Yes & Yes & Yes & Yes \\
\hline $\begin{array}{l}\text { Previous drugs } \\
\text { exposure }\end{array}$ & $\begin{array}{l}\text { For pre } \\
\text { existing } \\
\text { conditions, } \\
\text { Pregnancy } \\
\text { related }\end{array}$ & $\begin{array}{l}\text { Pregnancy } \\
\text { related }\end{array}$ & $\begin{array}{l}\text { For pre } \\
\text { existing } \\
\text { conditions, } \\
\text { Pregnancy } \\
\text { related }\end{array}$ & $\begin{array}{l}\text { Pregnancy } \\
\text { related }\end{array}$ & $\begin{array}{l}\text { Pregnancy } \\
\text { related }\end{array}$ & $\begin{array}{l}\text { Pregnancy } \\
\text { related }\end{array}$ & $\begin{array}{l}\text { For pre existing } \\
\text { conditions, } \\
\text { Pregnancy related }\end{array}$ & $\begin{array}{l}\text { For pre } \\
\text { existing } \\
\text { conditions, } \\
\text { Pregnancy } \\
\text { related }\end{array}$ \\
\hline $\begin{array}{l}\text { Drugs for } \\
\text { preexisting } \\
\text { diseases, which? }\end{array}$ & Antiretroviral & $\begin{array}{l}\text { Not } \\
\text { applicable }\end{array}$ & Antimalarial & Not applicable & $\begin{array}{l}\text { Not } \\
\text { applicable }\end{array}$ & $\begin{array}{l}\text { Not } \\
\text { applicable }\end{array}$ & $\begin{array}{l}\text { Antiepileptic, } \\
\text { Antiretroviral, } \\
\text { Analgesics, } \\
\text { Antituberculosis, } \\
\text { Neuropsychiatric, } \\
\text { Antibiotics }\end{array}$ & Antiretroviral \\
\hline $\begin{array}{l}\text { Pregnancy related } \\
\text { drugs, which? }\end{array}$ & $\begin{array}{l}\text { Iron, Folic } \\
\text { acid }\end{array}$ & $\begin{array}{l}\text { Iron, } \\
\text { Vitamins, } \\
\text { Calcium, } \\
\text { Antibiotics, } \\
\text { Misoprostol }\end{array}$ & $\begin{array}{l}\text { Iron, Folic } \\
\text { acid, Vitamins }\end{array}$ & Iron, Folic acid & $\begin{array}{l}\text { Iron, Folic } \\
\text { acid }\end{array}$ & $\begin{array}{l}\text { Iron, Folic } \\
\text { acid, } \\
\text { Corticoids, } \\
\text { Magnesium } \\
\text { sulphate }\end{array}$ & $\begin{array}{l}\text { Iron, Folic acid, } \\
\text { Vitamins }\end{array}$ & $\begin{array}{l}\text { Iron, } \\
\text { Vitamins, } \\
\text { Calcium }\end{array}$ \\
\hline $\begin{array}{l}\text { Vaccines exposure } \\
\text { registries }\end{array}$ & $\begin{array}{l}\text { Pregnancy } \\
\text { related, } \\
\text { Other }\end{array}$ & $\begin{array}{l}\text { Pregnancy } \\
\text { related }\end{array}$ & $\begin{array}{l}\text { Pregnancy } \\
\text { related, Other }\end{array}$ & $\begin{array}{l}\text { Pregnancy } \\
\text { related }\end{array}$ & $\begin{array}{l}\text { Pregnancy } \\
\text { related, } \\
\text { Other }\end{array}$ & $\begin{array}{l}\text { Pregnancy } \\
\text { related, } \\
\text { Other }\end{array}$ & Pregnancy related & N/A \\
\hline Vaccines, which & $\begin{array}{l}\text { Influenza, } \\
\text { Tetanus }\end{array}$ & $\begin{array}{l}\text { Influenza, } \\
\text { Tetanus }\end{array}$ & $\begin{array}{l}\text { Influenza, } \\
\text { Haemophilus } \\
\text { influenzae } \\
\text { type B, Other }\end{array}$ & Tetanus & $\begin{array}{l}\text { Tetanus, } \\
\text { Hepatitis B, } \\
\text { BCG, } \\
\text { Pentavalent }\end{array}$ & $\begin{array}{l}\text { Influenza, } \\
\text { Tetanus, } \\
\text { Hepatitis B }\end{array}$ & $\begin{array}{l}\text { Group B } \\
\text { Streptococcus }\end{array}$ & N/A \\
\hline
\end{tabular}

women to enter information directly into the system through their smartphones. OpenSRP promotes a mobile health platform that allows health workers to register and track patient data [167].

GN-MNHR, INDEPTH, PER/BDS and SIP coordinated the data collection and validation across the sites [17, 103, 161, 182]. In contrast, DHIS 2, OpenMRS , OpenSRP and SmartCare offered a module and platform that each site can customize, modify, and adapt for use with total autonomy [131, 152, 170, 178].

The tenth revision of the International Classification of Diseases (ICD10) was used to code outcomes and conditions by more than half of the systems: DHIS 2 [130, 131], INDEPTH [100], OpenMRS [154], SIP [161] and PER/BDS [176]. No information was found regarding how OpenSRP and SmartCare systems classified and coded outcomes. The GN-MNHR system does not use any standardized classification. Only INDEPTH had been used for phase IV safety trials and post-marketing surveillance by a maternal health research platform $[17,183]$.

\section{Data sources (Table 7)}

All the systems can collect patient data and longitudinally track pregnant women' progress and their babies 
Table 8 Maternal outcomes

\begin{tabular}{|c|c|c|c|c|c|c|c|c|}
\hline Variable & 1: DHIS2 & 2: GN MNHR & 3: INDEPTH & 4: OpenMRS & 5: OpenSRP & 6: SIP & 7: PER/BDS & 8: SmartCare \\
\hline Maternal death & Yes & Yes & Yes & Yes & Yes & Yes & Yes & Yes \\
\hline Preeclamsia/Eclampsia & Yes & Yes & No & Yes & N/A & Yes & N/A & $\mathrm{N} / \mathrm{A}$ \\
\hline Gestational hypertension & N/A & Yes & Yes & N/A & N/A & N/A & N/A & $\mathrm{N} / \mathrm{A}$ \\
\hline Foetal distress & No & No & Yes & Yes & Yes & Yes & Yes & N/A \\
\hline Ectopic pregnancy & No & Yes & No & N/A & N/A & N/A & N/A & N/A \\
\hline Postpartum hemorrhage & Yes & Yes & Yes & Yes & Yes & Yes & Yes & N/A \\
\hline Spontaneous Abortion & Yes & Yes & Yes & Yes & Yes & Yes & Yes & N/A \\
\hline Antenatal bleeding & No & Yes & Yes & Yes & Yes & Yes & Yes & N/A \\
\hline Dysfunctional labour & Yes & Yes & No & Yes & Yes & Yes & Yes & N/A \\
\hline Foetal growth retardation & Yes & No & No & Yes & N/A & Yes & Yes & $\mathrm{N} / \mathrm{A}$ \\
\hline Gestational diabetes & Yes & No & No & No & Yes & Yes & N/A & N/A \\
\hline Post-partum endometritis & N/A & Yes & No & No & Yes & Yes & N/A & $\mathrm{N} / \mathrm{A}$ \\
\hline Chorioamnionitis & N/A & No & No & No & Yes & Yes & N/A & $\mathrm{N} / \mathrm{A}$ \\
\hline $\begin{array}{l}\text { Premature preterm rupture of } \\
\text { membranes }\end{array}$ & No & No & No & N/A & N/A & N/A & N/A & N/A \\
\hline Preterm labor & No & No & No & N/A & N/A & N/A & N/A & N/A \\
\hline Insufficient cervix & No & No & No & N/A & N/A & N/A & N/A & $\mathrm{N} / \mathrm{A}$ \\
\hline
\end{tabular}

$\mathrm{N} / \mathrm{A}$ : Not available information

over the prenatal and postnatal periods. However, timing of capturing information from antenatal visits is different between the eight systems. GN-MNHR and INDEPTH collected antenatal care data retrospectively [17, 103]. GN-MNHR collected their data at enrollment and delivery [103], and INDEPTH collected past events by self-reported data from household visits [17].

Drug exposures during pregnancy were recorded widely (e.g., antimalarial and antiretroviral treatment, iron, folic acid and vitamins) $[17,68,103,114,127$,
150, 152, 165, 170, 178, 182]. Exposure to vaccines was also collected, mainly of certain vaccines related to pregnancy (Influenza, tetanus/pentavalent) as well as non-pregnancy related vaccines (Hepatitis B, BCG, Haemophilus influenzae type B) $[17,68,103,114$, 127, 130, 152, 165, 170, 178, 182]. PER/BDS system showed the widest drug and vaccine exposure recording, and intends to increase the list during the registry' s future national implementation [174, 175]. We did not find information about collecting this information for SmartCare.

Table 9 Maternal outcomes

\begin{tabular}{lllllllll}
\hline Variable & 1: DHIS2 & 2: GN MNHR & 3: INDEPTH & 4: OpenMRS & 5: OpenSRP & 6: SIP & 7: PER/BDS & 8: SmartCare \\
\hline Neonatal death & Yes & Yes & Yes & Yes & Yes & Yes & Yes & Yes \\
Congenital anomalies & Yes & Yes & Yes & No & Yes & Yes & Yes & N/A \\
Neonatal infections & Yes & Yes & Yes & No & Yes & Yes & Yes & N/A \\
Preterm birth & Yes & Yes & Yes & Yes & N/A & Yes & Yes & N/A \\
Stillbirth & Yes & Yes & Yes & Yes & N/A & Yes & Yes & N/A \\
Low birth weight & Yes & Yes & Yes & Yes & N/A & Yes & Yes & N/A \\
Small for gestational age & Yes & Yes & Yes & Yes & N/A & Yes & Yes & N/A \\
Neonatal encephalopathy & No & No & No & No & N/A & Yes & Yes & N/A \\
Respiratory distress & Yes & Yes & No & No & Yes & Yes & Yes & N/A \\
Failure to thrive & No & No & No & Yes & N/A & Yes & Yes & N/A \\
Microencephaly & No & No & No & No & Yes & Yes & Yes & N/A \\
Neonatal seizures & No & Yes & No & No & N/A & No & N/A & N/A \\
Neurodevelopmental delay & No & No & No & No & N/A & No & N/A & N/A \\
\hline
\end{tabular}

$\mathrm{N} / \mathrm{A}$ : Not available information 


\section{Maternal and neonatal outcomes (Tables 8 and 9)}

Twenty-nine $\mathrm{MNCH}$ outcomes in selected data collection systems were searched: 16 maternal outcomes and 13 neonatal outcomes. We did not find information about SmartCare regarding their recorded perinatal outcomes.

All systems collected vital data such as maternal and neonatal deaths. The most frequently recorded perinatal outcomes were fetal distress, postpartum hemorrhage, antenatal bleeding, dysfunctional labor, spontaneous abortion, congenital anomalies, neonatal infections, preterm birth, stillbirth, low birth weight, small for gestational age and respiratory distress. Some outcomes were not recorded by any of the selected systems, i.e., premature preterm rupture of membranes, preterm labor, insufficient cervix and neurodevelopmental delay [184].

The seven systems with available data recorded 13 to 22 perinatal outcomes out of a total of 29 perinatal outcomes. Of the 16 maternal outcomes evaluated, SIP $[161,163,165]$ and GN-MNHR [108, 110, 113, 119, 122] registered more than $50 \%$ of outcomes $(n=11$ and $n=10$ respectively), DHIS 2 [131, 142, 150], OpenMRS [154] and OpenSRP [168-170] registered 50\% of outcomes $(\mathrm{n}=8$ each) and INDEPTH $[38,46,49,68,77,100]$ and PER/BDS [182] less than $50 \%$ of outcomes ( $n=6$ each).

Of the 13 neonatal outcomes evaluated, SIP [161, 165], PER/BDS [175], GN-MNHR [105, 106, 108, 113, 114, 119], DHIS 2 [138, 145, 146, 150] and INDEPHT [38, $46,49,56,68,100]$ registered more than $50 \%,(n=11, n=$ $11, \mathrm{n}=10, \mathrm{n}=9$ and $\mathrm{n}=7$ respectively) and OpenMRS $[152,154]$ and OpenSRP $[169,170]$ less than $50 \%(\mathrm{n}=6$ and $n=5$ outcomes, respectively).

\section{Data quality (Table 10)}

This domain was evaluated by examining information on both external and internal quality control mechanisms used by data collection systems. Internal monitoring was the most frequently cited procedure, specifically preprogrammed checks to avoid incorrect data entry [29, 38, 103, 139, 152, 156, 170]. Regarding external monitoring, only half of the systems reported having the necessary structures to be subject to frequent auditing and manual reporting $[28,38,45,108,113,145]$. Only three systems demonstrated internal and external quality controls (DHIS 2, GN-MNHR and INDEPTH).

\section{Discussion}

Through the present scoping review, 8,069 records were screened, and eight active data collection systems were identified. These systems continuously collect individual maternal and neonatal data in LMICs that can be leveraged for active safety surveillance of novel maternal vaccines.

Among the eight systems, seven systems are being used in countries in Africa, four in Asia and three in Latin America. Data collection systems served as research networks, perinatal electronic medical records, or a custom software platform for health information systems. The eight data collection systems showed variability regarding their governance, system design, data management, data sources, outcomes collected and data quality. Among these systems, all except SIP protected privacy of the information collected through anonymization of data. All systems except for GN-MNHR and SIP demonstrated interoperability capabilities and used webbased platforms. Data were recorded from antenatal care to postnatal period in all systems; however, GN-MNHR and INDEPTH collected antenatal visits data retrospectively. All systems collected vital data such as maternal and neonatal deaths as well as recorded exposure to vaccines and drugs during pregnancy. The most frequently

Table 10 Data Quality

\begin{tabular}{|c|c|c|c|c|c|c|c|c|}
\hline Variable & 1: DHIS2 & 2: GN MNHR & 3: INDEPTH & 4: OpenMRS & 5: OpenSRP & 6: SIP & 7: PER/BDS & 8: SmartCare \\
\hline $\begin{array}{l}\text { External } \\
\text { monitoring } \\
\text { for data } \\
\text { quality }\end{array}$ & Yes & Yes & Yes & N/A & N/A & No & N/A & Yes \\
\hline $\begin{array}{l}\text { If YES- } \\
\text { Description } \\
\text { of the } \\
\text { mechanism/ } \\
\text { Process }\end{array}$ & $\begin{array}{l}\text { Weekly, } \\
\text { monthly audits }\end{array}$ & $\begin{array}{l}\text { Periodic } \\
\text { audits, } \\
\text { Monthly } \\
\text { reposts }\end{array}$ & $\begin{array}{l}\text { Periodic audits, Bi- } \\
\text { weeky reports, Online } \\
\text { data dashboard for real- } \\
\text { time monitoring }\end{array}$ & $\begin{array}{l}\text { Not } \\
\text { applicable }\end{array}$ & $\begin{array}{l}\text { Not } \\
\text { applicable }\end{array}$ & $\begin{array}{l}\text { Not } \\
\text { applicable }\end{array}$ & $\begin{array}{l}\text { Not } \\
\text { applicable }\end{array}$ & $\begin{array}{l}\text { Periodic } \\
\text { source data } \\
\text { verification }\end{array}$ \\
\hline $\begin{array}{l}\text { Internal } \\
\text { monitoring } \\
\text { for data } \\
\text { quality }\end{array}$ & Yes & Yes & Yes & Yes & Yes & Yes & N/A & $\mathrm{N} / \mathrm{A}$ \\
\hline $\begin{array}{l}\text { If YES- } \\
\text { Description } \\
\text { of the } \\
\text { mechanism/ } \\
\text { Process }\end{array}$ & $\begin{array}{l}\text { Pre- } \\
\text { programmed } \\
\text { data quality } \\
\text { checks, Visual } \\
\text { verification }\end{array}$ & $\begin{array}{l}\text { Pre- } \\
\text { programmed } \\
\text { data quality } \\
\text { checks }\end{array}$ & $\begin{array}{l}\text { Pre-programmed data } \\
\text { quality checks }\end{array}$ & $\begin{array}{l}\text { Pre- } \\
\text { programmed } \\
\text { data quality } \\
\text { checks }\end{array}$ & $\mathrm{N} / \mathrm{A}$ & $\begin{array}{l}\text { Pre- } \\
\text { programmed } \\
\text { data quality } \\
\text { checks }\end{array}$ & $\begin{array}{l}\text { Not } \\
\text { applicable }\end{array}$ & $\begin{array}{l}\text { Not } \\
\text { applicable }\end{array}$ \\
\hline
\end{tabular}

$\mathrm{N} / \mathrm{A}$ : Not available information 
recorded perinatal outcomes were fetal distress, postpartum hemorrhage, antenatal bleeding, dysfunctional labor, spontaneous abortion, congenital anomalies, neonatal infections, preterm birth, stillbirth abortion, low birth weight, small for gestational age, respiratory distress and failure to thrive, with variability among the systems. Any of the selected systems did not record the outcomes premature preterm rupture of membranes, preterm labor, insufficient cervix, and neurodevelopmental delay. GNMNHR and INDEPTH coordinated the data collection and validation across their sites. In contrast, the rest of the systems offered a module and open-source platform that each site can customize, modify, and adapt for use with total autonomy. The tenth revision of the International Classification of Diseases (ICD10) was used for coding outcomes and conditions by DHIS 2, INDEPTH, OpenMRS, SIP and PER/BDS. No information was found on how outcomes and conditions were coded for the systems OpenSRP and SmartCare. As far as we know, GN-MNHR system does not use any standardized classification.

One close antecedent to our study is the work published by Froen et al., who, using WHO frameworks, mapped electronic registries (eRegistries) for maternal and child health [185]. The authors conducted a webbased survey of public health officials in LMICs and a search of literature from 2005 to 2015 to assess country capacity, quality and data usage in reproductive health registries. Froen et al. found 32 paper and electronic registry systems in 23 countries, supporting commonly used electronic and mobile applications for health. During those years, countries were in transition from paperbased data collection to electronic systems but very few have integrated electronic backbone systems. A more detailed framework was used to assess and describe the existing and eligible attributes of $\mathrm{MNCH}$ health systems, focusing on electronic data collection systems [12]. Our broad search conducted in August 2019 identified three times the number of registries $(n=96)$. In contrast with their findings, we assessed that only eight were proficient in informing active safety vaccine surveillance system.

Zuber and colleagues [7] created a map of $\mathrm{MNCH}$ initiatives that collected health information to monitor maternal and child interventions in LMICs. The reported programs collected maternal and child health aggregate data and were fragmented in governance and financing and were duplicated in several related initiatives. They could not link individual-level data from pregnant women and their offspring including the linkage across individual records and multiple registers and sources. An active safety surveillance system for maternal vaccines would require statistics and monitoring of health data that reflect mother-baby dyad, characteristics, conditions and events from pregnancy to childbirth and postpartum care collected systematically, longitudinal, individual and uniform way. Our findings demonstrate that there are at least eight existing types of perinatal data collection systems/platforms implemented successfully in LMICS and can scale-up and collect $\mathrm{MNCH}$ individual-level data that track mothers and their babies.

Post-marketing surveillance of drugs used during pregnancy have been carried out in LMICs. Particularly, during the dolutegravir surveillance in Botswana (2018), 134 congenital abnormalities were identified in pregnant women exposed to this drug. Of these, the majority (104 cases) came from post-marketing studies, and only a few from spontaneous reports. However, although reporting post-marketing surveillance is useful, it lacks the ability to calculate prevalence rates because the true denominator is not usually available and births without defects are also underreported in LMICs frequently [186]. Another example, the International Maternal Pediatric Adolescents AIDS Clinical Trials (IMPAACT) network has been conducting clinical trials of drugs used during pregnancy with the aim of reducing perinatal transmission of human immunodeficiency virus. Some of them were phase IV trials and have provided important information during post-marketing stage. However, as limitation of those studies and as with most clinical trials data collection systems, some conclusions obtained might not be entirely extrapolated to the real world, and very low incidence adverse effects might not be detected in them [187]. Concerning antimalarial surveillance, a prospective observational study using HDSS (INDEPTH system) conducted in Burkina Faso, Kenya and Mozambique has evaluated artemisinin exposure and monitoring in pregnant women. Although the methods described and used in this study have been relevant in the development of pharmacovigilance of drugs in pregnancy and baseline perinatal prevalence rates might be measured in the regions, they have had certain limitations in quality and feasibility to collect certain outcomes. With the exception of the Kenya site where active surveillance has been carried out, in the other sites it was not possible to detect early miscarriages as well as the early identification of pregnancy [98].

The main strengths of our study are that we followed established methods [9, 188], utilized an exhaustive search strategy that included an in-depth grey literature search, and consulted large group of experts in the field with experience in pharmacovigilance, vaccine safety monitoring, as well as MNCH in LMICs on the results of this review.

The study's main limitations are the heterogeneous and incomplete available reporting, forcing us to look for multiple non-peer-reviewed reports and directly contact authors and data system authorities to obtain a 
complete picture of each system. On-site visits and interviews to key referents could improve the completeness of this information, although these methods were beyond the scope of our protocol. For example, quality of data, capacity for data sharing and prevalence of maternal and infant health outcomes in each site from each system could also be obtained during future site visits in order to supplement the findings of this review.

Another limitation of our study is related to the definitions of maternal and neonatal outcomes that each system reported as being measured. The GAIA case definitions were used as a guide in order to ensure extracting all relevant perinatal data outcomes. Due to the fact that many of these GAIA definitions are really complex and require a lot of information to be considered as correctly defined, we cannot affirm that the full case definitions in each system comply exactly with the parameters proposed by GAIA [184].

Our findings have important implications not only for safety surveillance in maternal vaccines but also for policymakers and other stakeholders committed to research in $\mathrm{MNCH}$. The analytical framework used demonstrated that all of the data collection systems identified in LMICs showed strengths and weaknesses to varying extents. However, several of the data collection systems are ready to inform future active safety surveillance. Regarding data protection, although most LMICs have not adopted a specific legislation or a Data Protection $\mathrm{Au}$ thority [15], seven out of eight systems in this review included an appropriate data protection process to protect personal information about women and their children to be used, intentionally or otherwise, for purposes other than understanding and informing the prevention of poor health outcomes or to measure the safety of vaccines. Variability in case definitions and diagnostic criteria across data sources, and among differing cultures and languages was presented as a challenge by experts. Lack of harmonization of case confirmation/classification among systems was also a defined problem in LMICs [7, 189]. However, among the eight data collection systems found in our review, seven used the International Classification of Diseases (ICD) codes for standardized reporting of diseases. This is a promising finding to advance the integration and harmonization of the collection of MNCH data across systems in LMICs. Further in-depth exploration of these systems will provide more details about their capacity.

Our work could help to recognize and overcome the highlighted knowledge gap regarding the existence and capacity of surveillance platforms in LMICs for novel maternal vaccines. Identifying individual $\mathrm{MNCH}$ data platforms for pregnancy and disease surveillance is the first key action needed to identify potential sentinel sites for implementing integrated active surveillance successfully.

\section{Conclusion}

We present a list of existing $\mathrm{MNCH}$ data capture systems in LMICs and describe in detail their characteristics, outreach, strengths, and limitations. This knowledge could potentially help policymakers, vaccine developers, researchers, and regulators to understand where to obtain population-based high-quality information on outcomes to inform and improve the conduct of vaccine active safety surveillance in LMICs.

\section{Abbreviations}

LMICS: Low- and middle-income countries; MNCH: Maternal neonatal and child health; GAIA: Global Alignment of Immunization Safety Assessment in pregnancy; WHO: World Health Organization; PRISMA: Preferred Reporting Items for Systematic Reviews and Meta-Analyses; GAVl: Global Alliance for Vaccines and Immunization; GN-MNHR: Global Network's Maternal Newborn Health Registry; INDEPTH: International Network for Demographic Evaluation of Populations and their Health; SIP: Perinatal Informatic System; PER/ BDS: Pregnancy Exposure Registry \& Birth Defects Surveillance;

OpenMRS: Open Medical Record System; OpenSRP: Open Smart Register Platform; DHIS 2: District Health Information Software 2; ICD 10: The tenth revision of the International Classification of Diseases

\section{Supplementary Information}

The online version contains supplementary material available at https://doi. org/10.1186/s12884-021-03686-9.

Additional file 1:. PRISMA Checklist. It contains the PRISMA Checklist.

Additional file 2:. Search strategy. It contains the search strategies used.

Additional file 3:. Data collection systems excluded. It contains the description of the excluded data collection systems.

\section{Acknowledgements}

The authors would like to thank Andrea Meyer and Sarah Matthews for English editing. We could not have done this study without the

collaboration and dedication of the Scoping Review Collaboration Group (author names and affiliation): Judith Absalon, MD, MPH (Pfizer, Inc); Steve Anderson, PhD, MPP (US Food \& Drug Administration); Fernando Althabe, MD (World Health Organization); Shabir Madhi, MBBCh, FCPaeds, PhD (University of the Witwatersrand); Elizabeth McClure, PhD (Research Triangle Institute; University of North Carolina at Chapel Hill); Flor M. Munoz, MD, MSc (Baylor College of Medicine); Kissa W. Mwamwitwa, Mpharm (Tanzania Medicines \& Medical Devices Authority); Annettee Nakimuli, MD, MMed Obs\&gyn, PhD (Kaiser Permanente Washington Health Research Institute); Jennifer Clark Nelson, PhD (Makerere University); Lisa Noguchi, PhD, CNM (Johns Hopkins University/Jhpiego); Lakshmi Panagiotakopoulos, MD, MPH (Centers for Disease Control and Prevention); Esperanca Sevene, MD, MSc, PhD (Eduardo Mondlane University); Patrick Zuber, MD (World Health Organization); Maria Belizan, MSC (Institute for Clinical Effectiveness and Health Policy); Eduardo Bergel, PhD (Institute for Clinical Effectiveness and Health Policy); Alvaro Ciganda, BSCS (Institute for Clinical Effectiveness and Health Policy); Daniel Comande, BSc (Institute for Clinical Effectiveness and Health Policy); and Veronica Pingray, MPH (Institute for Clinical Effectiveness and Health Policy).

\section{Authors' contributions}

MB: Conceptualization, Investigation, Methodology, Project administration, Supervision, Writing, Review and Editing. AC: Methodology, Project administration, Supervision, Writing, Review and Editing. AB: Methodology, Project administration, Supervision, Writing, Review and Editing. FRC: Investigation, Methodology, Writing, Review and Editing. FC: Investigation, Methodology, Writing, Review and Editing. XX: Conceptualization, Methodology, Writing, Review and Editing. AS: Conceptualization, Methodology, Writing, Review and Editing. SZ: Investigation, Methodology, Writing, Review and Editing. ASM: Conceptualization, Methodology, Writing, Review and Editing. PB: Conceptualization, Funding Acquisition, 
Methodology, Project administration, Supervision, Writing, Review and Editing. Scoping Review Collaboration Group: Methodology, Writing, Review and Editing. All authors have approved the submitted version.

\section{Funding}

This work was funded by Bill \& Melinda Gates Foundation (BMGF) (INV008443). The funding agency had no role in the final decision to submit the manuscript. Ajoke Sobanjo-ter Meulen (author affiliation: Bill \& Melinda Gates Foundation) provided feedback on Conceptualization, Methodology, Writing, Review and Editing.

\section{Availability of data and materials}

All data generated or analyzed during this study are included in this published article and its supplementary information files.

\section{Declarations}

Ethics approval and consent to participate

Not applicable since this is a secondary research.

\section{Consent for publication}

Not applicable

\section{Competing interests}

The authors take sole responsibility for the writing and content of the paper. All authors have nothing to disclose.

\section{Author details}

'Instituto de Efectividad Clínica y Sanitaria (IECS), Dr. Emilio Ravignani 2024 (C1014CPV), Buenos Aires, Argentina. ${ }^{2}$ Tulane University School of Public Health and Tropical Medicine, New Orleans, LA 70112, USA. ${ }^{3}$ University of Washington, Seattle, WA 98195-7631, USA. ${ }^{4}$ Bill \& Melinda Gates Foundation, Seattle, WA, USA.

Received: 10 November 2020 Accepted: 1 March 2021

Published online: 17 March 2021

\section{References}

1. GAPPS. Maternal Immunization Safety Monitoring In Low- and-Middleincome Countries: A Roadmap For Program Development. Global Alliance to Prevent Prematurity and Stillbirth. 2017. https://www.gapps.org/PDF/Ma ternallmmunizationSafetyMonitoringInLMICs.pdf. Accessed 19 May 2020.

2. Lackritz E, Stepanchak M: Maternal Immunization Safety Monitoring in Lowand Middle-Income Countries: A Roadmap for Program Development. 2017 https://www.gapps.org/PDF/MaternallmmunizationSafetyMonitoringInLMICs. pdf. Accessed 3 July 2020.

3. Engmann C, Fleming JA, Khan S, Innis BL, Smith JM, Hombach J, SobanjoTer Meulen A. Closer and closer? Maternal immunization: current promise, future horizons. J Perinatol. 2020;40(6):844-57.

4. Jones CE, Munoz FM, Spiegel HM, Heininger U, Zuber PL, Edwards KM, Lambach P, Neels P, Kohl KS, Gidudu J, et al. Guideline for collection, analysis and presentation of safety data in clinical trials of vaccines in pregnant women. Vaccine. 2016;34(49):5998-6006.

5. Kochhar S, Bonhoeffer J, Jones CE, Muñoz FM, Honrado A, Bauwens J, Sobanjo-Ter Meulen A, Hirschfeld S. Immunization in pregnancy clinical research in low- and middle-income countries - Study design, regulatory and safety considerations. Vaccine. 2017;35(48 Pt A):6575-81.

6. MCSP: What Data on Maternal and Newborn Health Do National Health Management Information Systems Include? 2018. https:/www.mcsprogram.org/. Accesed 20 July 2020

7. Zuber PLF, Moran AC, Chou D, Renaud F, Halleux C, Peña-Rosas JP, Viswanathan K, Lackritz E, Jakob R, Mason E, et al. Mapping the landscape of global programmes to evaluate health interventions in pregnancy: the need for harmonised approaches, standards and tools. BMJ Glob Health. 2018, 3(5):e001053.

8. Sobanjo-Ter Meulen A, Munoz FM, Kaslow DC, Klugman KP, Omer SB, Vora $P$, Stergachis A. Maternal interventions vigilance harmonization in low- and middle-income countries: Stakeholder meeting report; Amsterdam, May 1-2, 2018. Vaccine. 2019;37(20):2643-50.

9. Arksey H, O'Malley L. Scoping studies: towards a methodological framework. Int J Soc Res Methodol. 2005;8(1):19-32.
10. Tricco AC, Lillie E, Zarin W, O'Brien KK, Colquhoun H, Levac D, Moher D, Peters MDJ, Horsley T, Weeks L, et al. PRISMA Extension for Scoping Reviews (PRISMA-SCR): Checklist and Explanation. Ann Intern Med. 2018;169(7):467-73.

11. Berrueta M, Bardach A, Ciaponni A et al. Maternal and neonatal data collection systems in low- and middle-income countries: scoping review protocol [version 1; peer review: 3 approved]. Gates Open Res. 2020:4:18.

12. MEASURE Evaluation. Strengthening Health Information Systems in Lowand Middle-Income Countries A Model to Frame What We Know and What We Need to Learn. Chapel Hill: MEASURE Evaluation; 2019.

13. Health Metrics N, World Health O. Framework and standards for country health information systems. 2nd ed. Geneva: World Health Organization; 2008. https://apps.who.int/iris/handle/10665/43872. Accessed 1 June 2020

14. Dawne W: Model of a Community-Based Information System: Essential Components and Functions. 2018. https://www.measureevaluation.org/. Accessed 20 May 2020.

15. Commission Nationale de l'Informatique et des Libertés. Data protection around the world. 2020. https://www.cnil.fr/en/data-protection-around-theworld. Accessed 19 June 2020.

16. Monitoring and assessing the impact of vaccination and other childhood interventions for both boys and girls. 2017. https://dfcentre.com/wpcontent/uploads/2017/07/09-105SSI-App-3b-Policy-Brief.pdf. Accessed 19 June 2020.

17. INDEPTH Network. http://www.indepth-network.org/. Accessed 20 May 2020.

18. Bardaji A, Tembe N, Mucavele H, Massora S, Aguado T, Bassat Q, Macete E, Menéndez C: Research capacities at ISGlobal to conduct vaccine trials and clinical studies in support to maternal immunization research in Mozambique. In: 5th International Neonatal \& Maternal Immunization Symposium. Vancouver; 2019.

19. Dure Technology: Overview of the nutrition information system in Lao PDR. In. Montpellier: Agropolis International. 2019. http://www.nipn-nutrition-pla tforms.org/. Accessed 19 June 2020.

20. Duthe G: The impact of the marital status of the mother at birth on the mortality risks during childhood in rural Senegal: a gender perspective. In: PAA Annual meeting. Chicago; 2017.

21. Haile D, Kondale M, Andarge E, Tunje A, Fikadu T, Boti N. Level of completion along continuum of care for maternal and child health services and factors associated with it among women in Arba Minch Zuria Woreda, Gamo Zone, Southern Ethiopia: a community based cross-sectional study. PLoS One. 2020;15(6):e0221670.

22. Lankoande YB, Pison G: Does pregnancy follow up improve reliability of under five mortality estimates in Health and Demographic Surveillance Systems? Insights from Bandafassi and Niakhar (Senegal). In: 8th African Population Conference. Entebbe; 2019. http://uaps2019.popconf.org/abstra cts/190752.

23. Nurul A, Chowdhury H, Das S, Ali A, Streatfield P. Causes of death in two rural demographic surveillance sites in Bangladesh, 2004-2010: automated coding of verbal autopsies using InterVA-4. (Special Issue: INDEPTH network cause-specific mortality.). Global Health Action. 2014;7:25511 32 ref 2014

24. Olatunji A, Doctor HV. The Potential Role of a Health and Demographic Surveillance System in Rural Northern Nigeria to Reduce Maternal and Child Deaths. Health. 2014;7(12):1741-6.

25. Afework MF, Gebregiorgis SH, Roro MA, Lemma AM, Ahmed S. Do Health and Demographic Surveillance Systems benefit local populations? Maternal care utilisation in Butajira HDSS, Ethiopia. Glob Health Action. 2014;7:24228.

26. Alabi O, Doctor HV, Afenyadu GY, Findley SE. Lessons learned from setting up the Nahuche Health and Demographic Surveillance System in the resourceconstrained context of northern Nigeria. Glob Health Action. 2014;7: 23368.

27. Alabi O, Doctor HV, Jumare A, Sahabi N, Abdulwahab A, Findley SE, Abubakar SD. Health \& demographic surveillance system profile: the Nahuche Health and Demographic Surveillance System, Northern Nigeria (Nahuche HDSS). Int J Epidemiol. 2014;43(6):1770-80.

28. Alam N, Ali T, Razzaque A, Rahman M, Zahirul Haq M, Saha SK, Ahmed A, Sarder AM, Moinuddin Haider M, Yunus M, et al. Health and Demographic Surveillance System (HDSS) in Matlab, Bangladesh. Int J Epidemiol. 2017; 46(3):809-16.

29. Alam N, Townend J. The neighbourhood method for measuring differences in maternal mortality, infant mortality and other rare demographic events. Plos One. 2014;9(1):e83590.

30. Alberts M, Dikotope SA, Choma SR, Masemola ML, Modjadji SE, Mashinya F, Burger S, Cook I, Brits SJ, Byass P. Health \& Demographic Surveillance 
System Profile: The Dikgale Health and Demographic Surveillance System. Int J Epidemiol. 2015:44(5):1565-71.

31. Amek NO, Odhiambo FO, Khagayi S, Moige H, Orwa G, Hamel MJ, Van Eijk A, Vulule J, Slutsker L, Laserson KF. Childhood cause-specific mortality in rural Western Kenya: application of the InterVA-4 model. Glob Health Action. 2014;7:25581.

32. Aponte JJ, Aide P, Renom M, Mandomando I, Bassat Q, Sacarlal J, Manaca MN, Lafuente S, Barbosa A, Leach A, et al. Safety of the RTS,S/ASO2D candidate malaria vaccine in infants living in a highly endemic area of Mozambique: a double blind randomised controlled phase I/llb trial. Lancet. 2007;370(9598):1543-51.

33. Arikpo I, Okoro A, Esu E, Aquaisua E, Ekinya I, Meremikwu M. Differences in Population Dynamics and Uptake of Reproductive Health Services in the Urban and Rural Cohorts of Cross River Health and Demographic Surveillance System of Southern Nigeria. Developing Country Studies. 2019; 9:64-71.

34. Arnaldo P, Rovira-Vallbona E, Langa JS, Salvador C, Guetens P, Chiheb D, Xavier B, Kestens L, Enosse SM, Rosanas-Urgell A. Uptake of intermittent preventive treatment and pregnancy outcomes: health facilities and community surveys in Chókwè district, southern Mozambique. Malar J. 2018;17(1):109.

35. Assefa N, Lakew Y, Belay B, Kedir H, Zelalem D, Baraki N, Damena M, Oljira L, Ashenafi W, Dedefo M. Neonatal mortality and causes of death in Kersa Health and Demographic Surveillance System (Kersa HDSS), Ethiopia, 20082013. Matern Health Neonatol Perinatol. 2016;2:7.

36. Assefa N, Oljira L, Baraki N, Demena M, Zelalem D, Ashenafi W, Dedefo M. HDSS Profile: The Kersa Health and Demographic Surveillance System. Int Epidemiol. 2016;45(1):94-101.

37. Assefa N, Semahegn A. Fertility is below replacement in Harar Health and Demographic Surveillance System (Harar HDSS), Harar town, Eastern Ethiopia. Fertil Res Pract. 2016;2:10.

38. Baschieri A, Gordeev VS, Akuze J, Kwesiga D, Blencowe H, Cousens S, Waiswa P, Fisker AB, Thysen SM. Rodrigues A et al: "Every Newborn-INDE PTH" (EN-INDEPTH) study protocol for a randomised comparison of household survey modules for measuring stillbirths and neonatal deaths in five Health and Demographic Surveillance sites. J Glob Health. 2019;9(1): 010901

39. Bawah A, Houle B, Alam N, Razzaque A, Streatfield PK, Debpuur C, Welaga P, Oduro A, Hodgson A, Tollman S, et al. The Evolving Demographic and Health Transition in Four Low- and Middle-Income Countries: Evidence from Four Sites in the INDEPTH Network of Longitudinal Health and Demographic Surveillance Systems. Plos One. 2016;11(6):e0157281.

40. Becher H, Müller O, Dambach P, Gabrysch S, Niamba L, Sankoh O, Simboro S, Schoeps A, Stieglbauer G, Yé Y, et al. Decreasing child mortality, spatial clustering and decreasing disparity in North-Western Burkina Faso. Trop Med Int Health. 2016;21(4):546-55.

41. Bocoum FY, Tarnagda G, Bationo F, Savadogo JR, Nacro S, Kouanda S, Zarowsky C. Introducing onsite antenatal syphilis screening in Burkina Faso: implementation and evaluation of a feasibility intervention tailored to a local context. BMC Health Serv Res. 2017;17(1):378.

42. Bogale TN, Worku AG, Bikis GA, Kebede ZT. Why gone too soon? Examining social determinants of neonatal deaths in northwest Ethiopia using the three delay model approach. BMC Pediatr. 2017;17(1):216.

43. Coates MM, Kamanda M, Kintu A, Arikpo I, Chauque A, Mengesha MM, Price AJ, Sifuna P, Wamukoya M, Sacoor CN, et al. A comparison of all-cause and cause-specific mortality by household socioeconomic status across seven INDEPTH network health and demographic surveillance systems in subSaharan Africa. Glob Health Action. 2019;12(1):1608013.

44. Crampin AC, Dube A, Mboma S, Price A, Chihana M, Jahn A, Baschieri A, Molesworth A, Mwaiyeghele E, Branson K, et al. Profile: the Karonga Health and Demographic Surveillance System. Int J Epidemiol. 2012; 41(3):676-85.

45. Cunningham SA, Shaikh NI, Nhacolo A, Raghunathan PL, Kotloff K, Naser AM, Mengesha MM, Adedini SA, Misore T, Onuwchekwa UU, et al. Health and Demographic Surveillance Systems Within the Child Health and Mortality Prevention Surveillance Network. Clin Infect Dis. 2019;69(Suppl 4):S274-s279.

46. Delaunay V, Douillot L, Diallo A, Dione D, Trape JF, Medianikov O, Raoult D, Sokhna C. Profile: the Niakhar Health and Demographic Surveillance System. Int J Epidemiol. 2013;42(4):1002-11.

47. Deribew A, Ojal J, Karia B, Bauni E, Oteinde M. Under-five mortality rate variation between the Health and Demographic Surveillance System (HDSS) and Demographic and Health Survey (DHS) approaches. BMC Public Health. 2016;16(1):1118

48. Derra K, Rouamba E, Kazienga A, Ouedraogo S, Tahita MC, Sorgho H, Valea I, Tinto H. Profile: Nanoro Health and Demographic Surveillance System. Int J Epidemiol. 2012;41(5):1293-301.

49. Derso T, Biks GA, Tariku A, Tebeje NB, Gizaw Z, Muchie KF, Shimeka A, Kebede Y, Abebe SM, Yitayal M, et al. Correlates of early neonatal feeding practice in Dabat HDSS site, northwest Ethiopia. Int Breastfeed J. 2017;12:25.

50. Derso T, Tariku A, Biks GA, Wassie MM. Stunting, wasting and associated factors among children aged 6-24 months in Dabat health and demographic surveillance system site: A community based cross-sectional study in Ethiopia. BMC Pediatr. 2017;17(1):96.

51. Fekadu A, Yitayal M, Alemayehu GA, Abebe SM, Ayele TA, Tariku A, Andargie G, Teshome DF, Gelaye KA. Frequent Antenatal Care Visits Increase Institutional Delivery at Dabat Health and Demographic Surveillance System Site, Northwest Ethiopia. J Pregnancy. 2019;2019:1690986.

52. Geubbels E, Amri S, Levira F, Schellenberg J, Masanja H, Nathan R. Health \& Demographic Surveillance System Profile: The Ifakara Rural and Urban Health and Demographic Surveillance System (Ifakara HDSS). Int J Epidemiol. 2015;44(3):848-61.

53. Ghosh S, Barik A, Majumder S, Gorain A, Mukherjee S, Mazumdar S, Chatterjee K, Bhaumik SK, Bandyopadhyay SK, Satpathi B, et al. Health \& Demographic Surveillance System Profile: The Birbhum population project (Birbhum HDSS). Int J Epidemiol. 2015;44(1):98-107.

54. Gorain A, Barik A, Chowdhury A, Rai RK. Preference in place of delivery among rural Indian women. Plos One. 2017;12(12):e0190117.

55. Gyapong M, Sarpong D, Awini E, Manyeh AK, Tei D, Odonkor G, Agyepong IA, Mattah P, Wontuo P, Attaa-Pomaa M, et al. Profile: the Dodowa HDSS. Int J Epidemiol. 2013;42(6):1686-96.

56. Hazard RH, Alam N, Chowdhury HR, Adair T, Alam S, Streatfield PK, Riley ID, Lopez AD. Comparing tariff and medical assistant assigned causes of death from verbal autopsy interviews in Matlab, Bangladesh: implications for a health and demographic surveillance system. Popul Health Metr. 2018;16(1): 10.

57. Helleringer S, Pison G, Masquelier B, Kanté AM, Douillot L, Ndiaye CT, Duthé G, Sokhna C, Delaunay V. Improving survey data on pregnancy-related deaths in low-and middle-income countries: a validation study in Senegal. Trop Med Int Health. 2015;20(11):1415-23.

58. Herbst K, Juvekar S, Bhattacharjee T, Bangha M, Patharia N, Tei T, Gilbert B, Sankoh O. The INDEPTH Data Repository: An International Resource for Longitudinal Population and Health Data From Health and Demographic Surveillance Systems. J Empir Res Hum Res Ethics. 2015;10(3):324-33.

59. Jahn A, Floyd S, McGrath N, Crampin AC, Kachiwanda L, Mwinuka V, Zaba B, Fine PE, Glynn JR. Child mortality in rural Malawi: HIV closes the survival gap between the socio-economic strata. Plos One. 2010;5(6):e11320.

60. Jasseh M, Gomez P, Greenwood BM, Howie SR, Scott S, Snell PC, Bojang K, Cham M, Corrah T, D'Alessandro U. Health \& Demographic Surveillance System Profile: Farafenni Health and Demographic Surveillance System in The Gambia. Int J Epidemiol. 2015;44(3):837-47.

61. Kabudula CW, Houle B, Collinson MA, Kahn K, Gómez-Olivé FX, Tollman S, Clark SJ. Socioeconomic differences in mortality in the antiretroviral therapy era in Agincourt, rural South Africa, 2001-13: a population surveillance analysis. Lancet Glob Health. 2017;5(9):e924-35.

62. Kadobera D, Waiswa P, Peterson S, Blencowe H, Lawn J, Kerber K, Tumwesigye NM. Comparing performance of methods used to identify pregnant women, pregnancy outcomes, and child mortality in the IgangaMayuge Health and Demographic Surveillance Site, Uganda. Glob Health Action. 2017:10(1):1356641.

63. Kahn K, Collinson MA, Gómez-Olivé FX, Mokoena O, Twine R, Mee P, Afolabi SA, Clark BD, Kabudula CW, Khosa A, et al. Profile: Agincourt health and sociodemographic surveillance system. Int J Epidemiol. 2012;41(4):988-1001.

64. Kaneko S, K'Opiyo J, Kiche I, Wanyua S, Goto K, Tanaka J, Changoma M, Ndemwa M, Komazawa O, Karama M, et al. Health and Demographic Surveillance System in the Western and coastal areas of Kenya: an infrastructure for epidemiologic studies in Africa. J Epidemiol. 2012;22(3): 276-85.

65. Kant S, Misra P, Gupta S, Goswami K, Krishnan A, Nongkynrih B, Rai SK, Srivastava R, Pandav CS. The Ballabgarh Health and Demographic Surveillance System (CRHSP-AllMS). Int J Epidemiol. 2013;42(3):758-68.

66. Kishamawe C, Isingo R, Mtenga B, Zaba B, Todd J, Clark B, Changalucha J, Urassa M. Health \& Demographic Surveillance System Profile: The Magu 
Health and Demographic Surveillance System (Magu HDSS). Int J Epidemiol. 2015;44(6):1851-61.

67. Koné S, Baikoro N, N'Guessan Y, Jaeger FN, Silué KD, Fürst T, Hürlimann E, Ouattara M, Séka MC, N'Guessan NA, et al. Health \& Demographic Surveillance System Profile: The Taabo Health and Demographic Surveillance System, Côte d'Ivoire. Int J Epidemiol. 2015:44(1):87-97.

68. Koné S, Hürlimann E, Baikoro N, Dao D, Bonfoh B, N'Goran EK, Utzinger J, Jaeger FN. Pregnancy-related morbidity and risk factors for fatal foetal outcomes in the Taabo health and demographic surveillance system, Côte d'Ivoire. BMC Pregnancy Childbirth. 2018;18(1):216.

69. Kouanda S, Bado A, Yaméogo M, Nitièma J, Yaméogo G, Bocoum F, Millogo T, Ridde V, Haddad S, Sondo B. The Kaya HDSS, Burkina Faso: a platform for epidemiological studies and health programme evaluation. Int J Epidemiol. 2013;42(3):741-9.

70. Malaviya P, Picado A, Hasker E, Ostyn B, Kansal S, Singh RP, Shankar R, Boelaert M, Sundar S. Health \& Demographic Surveillance System profile: the Muzaffarpur-TMRC Health and Demographic Surveillance System. Int J Epidemiol. 2014;43(5):1450-7.

71. Manyeh AK, Amu A, Akpakli DE, Williams J, Gyapong M. Socioeconomic and demographic factors associated with caesarean section delivery in Southern Ghana: evidence from INDEPTH Network member site. BMC Pregnancy Childbirth. 2018;18(1):405

72. Marbán-Castro E, Sacoor C, Nhacolo A, Augusto O, Jamisse E, López-Varela E, Casellas A, Aponte JJ, Bassat Q, Sigauque B, et al. BCG vaccination in southern rural Mozambique: an overview of coverage and its determinants based on data from the demographic and health surveillance system in the district of Manhiça. BMC Pediatr. 2018;18(1):56.

73. Musa A, Assefa N, Weldegebreal F, Mitiku H, Teklemariam Z. Factor associated with experience of modern contraceptive use before pregnancy among women who gave birth in Kersa HDSS, Ethiopia. BMC Public Health. 2016;16:614.

74. Odhiambo FO, Laserson KF, Sewe M, Hamel MU, Feikin DR, Adazu K, Ogwang S, Obor D, Amek N, Bayoh N, et al. Profile: the KEMR//CDC Health and Demographic Surveillance System-Western Kenya. Int J Epidemiol. 2012;41(4):977-87.

75. Partap U, Young EH, Allotey P, Sandhu MS, Reidpath DD. Characterisation and correlates of stunting among Malaysian children and adolescents aged 6-19 years. Glob Health Epidemiol Genom. 2019;4:e2.

76. Partap U, Young EH, Allotey P, Soyiri IN, Jahan N, Komahan K, Devarajan N, Sandhu MS, Reidpath DD. HDSS Profile: The South East Asia Community Observatory Health and Demographic Surveillance System (SEACO HDSS). Int J Epidemiol. 2017;46(5):1370-1371g.

77. Pison G, Beck B, Ndiaye O, Diouf PN, Senghor P, Duthé G, Fleury L, Sokhna C, Delaunay V. HDSS Profile: Mlomp Health and Demographic Surveillance System (Mlomp HDSS), Senegal. Int J Epidemiol. 2018;47(4):1025-33.

78. Pison G, Douillot L, Kante AM, Ndiaye O, Diouf PN, Senghor P, Sokhna C, Delaunay V. Health \& demographic surveillance system profile: Bandafassi Health and Demographic Surveillance System (Bandafassi HDSS), Senegal. Int J Epidemiol. 2014;43(3):739-48.

79. Price J, Willcox M, Kabudula CW, Herbst K, Kahn K, Harnden A. Home deaths of children under 5 years in rural South Africa: a population-based longitudinal study. Trop Med Int Health. 2019;24(7):862-78.

80. Rosário EVN, Costa D, Francisco D, Brito M. HDSS Profile: The Dande Health and Demographic Surveillance System (Dande HDSS, Angola). Int J Epidemiol. 2017:46(4):1094-1094g.

81. Rossier C, Muindi K, Soura A, Mberu B, Lankoande B, Kabiru C, Millogo R. Maternal health care utilization in Nairobi and Ouagadougou: evidence from HDSS. Glob Health Action. 2014;7:24351.

82. Rossier C, Soura A, Baya B, Compaoré G, Dabiré B, Dos Santos S, Duthé G, Gnoumou B, Kobiané JF, Kouanda S, et al. Profile: the Ouagadougou Health and Demographic Surveillance System. Int J Epidemiol. 2012;41(3): 658-66.

83. Sacoor C, Nhacolo A, Nhalungo D, Aponte JJ, Bassat Q, Augusto O, Mandomando I, Sacarlal J, Lauchande N, Sigaúque B, et al. Profile: Manhiça Health Research Centre (Manhiça HDSS). Int J Epidemiol. 2013;42(5):1309-18.

84. Sacoor C, Payne B, Augusto O, Vilanculo F, Nhacolo A, Vidler M, Makanga PT, Munguambe K, Lee T, Macete E, et al. Health and socio-demographic profile of women of reproductive age in rural communities of southern Mozambique. Plos One. 2018;13(2):e0184249.

85. Salzberg NT, Sivalogan K, Bassat Q, Taylor AW, Adedini S, El Arifeen S, Assefa N, Blau DM, Chawana R, Cain CJ, et al. Mortality Surveillance Methods to Identify and Characterize Deaths in Child Health and Mortality Prevention Surveillance Network Sites. Clin Infect Dis. 2019;69(Suppl 4):S262-s273.
86. Sankoh O, Byass P. Cause-specific mortality at INDEPTH Health and Demographic Surveillance System Sites in Africa and Asia: concluding synthesis. Glob Health Action. 2014;7:25590.

87. Sankoh O, Sharrow D, Herbst K, Whiteson Kabudula C, Alam N, Kant S, Ravn H, Bhuiya A, Thi Vui L, Darikwa T, et al. The INDEPTH standard population for low- and middle-income countries, 2013. Glob Health Action. 2014;7: 23286.

88. Scott JA, Bauni E, Moisi JC, Ojal J, Gatakaa H, Nyundo C, Molyneux CS, Kombe F, Tsofa B, Marsh K, et al. Profile: The Kilifi Health and Demographic Surveillance System (KHDSS). Int J Epidemiol. 2012;41(3):650-7.

89. Selemani M, Mwanyangala MA, Mrema S, Shamte A, Kajungu D, Mkopi A, Mahande MJ, Nathan R. The effect of mother's age and other related factors on neonatal survival associated with first and second birth in rural, Tanzania: evidence from Ifakara health and demographic surveillance system in rural Tanzania. BMC Pregnancy Childbirth. 2014;14:240.

90. Sié A, Louis VR, Gbangou A, Müller O, Niamba L, Stieglbauer G, Yé M, Kouyaté B, Sauerborn R, Becher $\mathrm{H}$. The Health and Demographic Surveillance System (HDSS) in Nouna, Burkina Faso, 1993-2007. Glob Health Action. 2010;3(1).

91. Sifuna P, Otieno L, Ogwang S, Ogutu B, Andagalu B, Owuoth J, Singoei V, Cowden J, Otieno W. Cause-specific mortality in the Kombewa health and demographic surveillance systems site, rural Western Kenya from 2011-2015. Glob Health Action. 2018;11(1):1442959.

92. Sifuna P, Oyugi M, Ogutu B, Andagalu B, Otieno A, Owira V, Otsyula N, Oyieko J, Cowden J, Otieno L, et al. Health \& demographic surveillance system profile: The Kombewa health and demographic surveillance system (Kombewa HDSS). Int J Epidemiol. 2014;43(4):1097-104.

93. Streatfield PK, Alam N, Compaoré Y, Rossier C, Soura AB, Bonfoh B, Jaeger F, Ngoran EK, Utzinger J, Gomez P, et al. Pregnancy-related mortality in Africa and Asia: evidence from INDEPTH Health and Demographic Surveillance System sites. Glob Health Action. 2014;7:25368.

94. Streatfield PK, Khan WA, Bhuiya A, Alam N, Sié A, Soura AB, Bonfoh B, Ngoran EK, Weldearegawi B, Jasseh M, et al. Cause-specific mortality in Africa and Asia: evidence from INDEPTH health and demographic surveillance system sites. Glob Health Action. 2014;7:25362.

95. Streatfield PK, Khan WA, Bhuiya A, Hanifi SM, Alam N, Ouattara M, Sanou A, Sié A, Lankoandé B, Soura AB, et al. Cause-specific childhood mortality in Africa and Asia: evidence from INDEPTH health and demographic surveillance system sites. Glob Health Action. 2014;7:25363.

96. Tesfaye G, Loxton D, Chojenta C, Assefa N, Smith R. Magnitude, trends and causes of maternal mortality among reproductive aged women in Kersa health and demographic surveillance system, eastern Ethiopia. BMC Womens Health. 2018;18(1):198.

97. Thysen SM, Fernandes M, Benn CS, Aaby P, Fisker AB. Cohort profile : Bandim Health Project's (BHP) rural Health and Demographic Surveillance System (HDSS)-a nationally representative HDSS in Guinea-Bissau. BMJ Open. 2019;9(6):e028775.

98. Tinto H, Sevene E, Dellicour S, Calip GS, d'Alessandro U, Macete E, Nakanabo-Diallo S, Kazienga A, Valea I, Sorgho H, et al. Assessment of the safety of antimalarial drug use during early pregnancy (ASAP): protocol for a multicenter prospective cohort study in Burkina Faso, Kenya and Mozambique. Reprod Health. 2015;12:112.

99. Tran TK, Nguyen $C T$, Nguyen HD, Eriksson B, Bondjers G, Gottvall K, Ascher H, Petzold M. Urban - rural disparities in antenatal care utilization: a study of two cohorts of pregnant women in Vietnam. BMC Health Serv Res. 2011;11: 120.

100. Waiswa P, Akuze J, Moyer C, Kwesiga D, Arthur S, Sankoh O, Welaga P, Bangha M, Eminas J, Muuo S, et al. Status of birth and pregnancy outcome capture in Health Demographic Surveillance Sites in 13 countries. Int J Public Health. 2019:64(6):909-20.

101. Wanyua S, Ndemwa M, Goto K, Tanaka J, K'Opiyo J, Okumu S, Diela P, Kaneko S, Karama M, Ichinose Y, et al. Profile: the Mbita health and demographic surveillance system. Int J Epidemiol. 2013;42(6):1678-85.

102. The Global Network for Women's and Children's Health Research. In. s.l.: Foundation for the National Institutes of Health; 2019. https://www.nichd. nih.gov/research/supported/globalnetwork. Accessed 20 May 2020.

103. RTI: Research Triangle Institute. https://www.rti.org/. Accessed 20 june 2020.

104. Goudar S, Goco N, Somannavar M, Vernekar S, Mallapur A, Moore J, Wallace D, Sloan N, Archana P, Hibberd P, et al. Institutional deliveries and perinatal and neonatal mortality in southern and central India. (Special Issue: Research reports from the NICHD Global Network for Women's and 
Children's Health Research Maternal and Newborn Health Registry.). Reprod Health. 2015;12(Suppl 2):S13 33 ref 2015.

105. Althabe F, Moore JL, Gibbons L, Berrueta M, Goudar SS, Chomba E, Derman RJ, Patel A, Saleem S, Pasha O, et al. Adverse maternal and perinatal outcomes in adolescent pregnancies: The Global Network's Maternal Newborn Health Registry study. Reprod Health. 2015;12(Suppl 2):S8.

106. Belizán JM, McClure EM, Goudar SS, Pasha O, Esamai F, Patel A, Chomba E, Garces A, Wright LL, Koso-Thomas M, et al. Neonatal death in low- to middle-income countries: a global network study. Am J Perinatol. 2012; 29(8):649-56.

107. Bellad MB, Vidler M, Honnungar NV, Mallapur A, Ramadurg U, Charanthimath U, Katageri G, Bannale S, Kavi A, Karadiguddi C, et al. Maternal and Newborn Health in Karnataka State, India: The Community Level Interventions for Pre-Eclampsia (CLIP) Trial's Baseline Study Results. Plos One. 2017;12(1):e0166623.

108. Bose CL, Bauserman M, Goldenberg RL, Goudar SS, McClure EM, Pasha O, Carlo WA, Garces A, Moore JL, Miodovnik M, et al. The Global Network Maternal Newborn Health Registry: a multi-national, community-based registry of pregnancy outcomes. Reprod Health. 2015;12(Suppl 2):S1.

109. Bucher S, Marete I, Tenge C, Liechty EA, Esamai F, Patel A, Goudar SS, Kodkany B, Garces A, Chomba E, et al. A prospective observational description of frequency and timing of antenatal care attendance and coverage of selected interventions from sites in Argentina, Guatemala, India, Kenya, Pakistan and Zambia. Reprod Health. 2015;12(Suppl 2):S12.

110. Dhaded SM, Somannavar MS, Vernekar SS, Goudar SS, Mwenche M, Derman R, Moore JL, Patel A, Pasha O, Esamai F, et al. Neonatal mortality and coverage of essential newborn interventions 2010 - 2013: a prospective, population-based study from low-middle income countries. Reprod Health. 2015;12(Suppl 2):S6.

111. Duffy CR, Moore IL, Saleem S, Tshefu A, Bose CL, Chomba E, Carlo WA, Garces AL, Krebs NF, Hambidge KM, et al. Malpresentation in low- and middle-income countries: Associations with perinatal and maternal outcomes in the Global Network. Acta Obstet Gynecol Scand. 2019;98(3):300-8.

112. Gisore P, Shipala E, Otieno K, Rono B, Marete I, Tenge C, Mabeya H, Bucher S, Moore J, Liechty E, et al. Community based weighing of newborns and use of mobile phones by village elders in rural settings in Kenya: a decentralised approach to health care provision. BMC Pregnancy Childbirth. 2012;12:15.

113. Goldenberg RL, McClure EM, Bose CL, Jobe AH, Belizán JM. Research results from a registry supporting efforts to improve maternal and child health in low and middle income countries. Reprod Health. 2015;12:54.

114. Goldenberg RL, Thorsten VR, Althabe F, Saleem S, Garces A, Carlo WA, Pasha O, Chomba E, Goudar S, Esamai F, et al. The global network antenatal corticosteroids trial: impact on stillbirth. Reprod Health. 2016;13(1):68.

115. Goudar SS, Goco N, Somannavar MS, Vernekar SS, Mallapur AA, Moore JL, Wallace DD, Sloan NL, Patel A, Hibberd PL, et al. Institutional deliveries and perinatal and neonatal mortality in Southern and Central India. Reprod Health. 2015;12(Suppl 2):S13.

116. Goudar SS, Stolka KB, Koso-Thomas M, Honnungar NV, Mastiholi SC, Ramadurg UY, Dhaded SM, Pasha O, Patel A, Esamai F, et al. Data quality monitoring and performance metrics of a prospective, population-based observational study of maternal and newborn health in low resource settings. Reprod Health. 2015;12(Suppl 2):S2.

117. Harrison MS, Ali S, Pasha O, Saleem S, Althabe F, Berrueta M, Mazzoni A, Chomba E, Carlo WA, Garces A, et al. A prospective population-based study of maternal, fetal, and neonatal outcomes in the setting of prolonged labor, obstructed labor and failure to progress in low- and middle-income countries. Reprod Health. 2015;12(Suppl 2):S9.

118. Kodkany BS, Derman RJ, Honnungar NV, Tyagi NK, Goudar SS, Mastiholi SC, Moore JL, McClure EM, Sloan N, Goldenberg RL. Establishment of a Maternal Newborn Health Registry in the Belgaum District of Karnataka, India. Reprod Health. 2015;12(Suppl 2):S3.

119. McClure EM, Garces A, Saleem S, Moore JL, Bose CL, Esamai F, Goudar SS, Chomba E, Mwenechanya M, Pasha O, et al. Global Network for Women's and Children's Health Research: probable causes of stillbirth in low- and middle-income countries using a prospectively defined classification system. BJOG. 2018;125(2):131-8.

120. McClure EM, Saleem S, Goudar SS, Moore JL, Garces A, Esamai F, Patel A, Chomba E, Althabe F, Pasha O, et al. Stillbirth rates in low-middle income countries 2010 - 2013: a population-based, multi-country study from the Global Network. Reprod Health. 2015;12(Suppl 2):S7.
121. Pasha O, Goudar SS, Patel A, Garces A, Esamai F, Chomba E, Moore JL, Kodkany BS, Saleem S, Derman RJ, et al. Postpartum contraceptive use and unmet need for family planning in five low-income countries. Reprod Health. 2015;12(Suppl 2):S11.

122. Pasha O, McClure EM, Saleem S, Tikmani SS, Lokangaka A, Tshefu A, Bose $\mathrm{CL}$, Bauserman M, Mwenechanya M, Chomba E, et al. A prospective cause of death classification system for maternal deaths in low and middleincome countries: results from the Global Network Maternal Newborn Health Registry. BJOG. 2018;125(9):1137-43.

123. Pasha O, Saleem S, Ali S, Goudar SS, Garces A, Esamai F, Patel A, Chomba E, Althabe $\mathrm{F}$, Moore $\mathrm{J}$, et al. Maternal and newborn outcomes in Pakistan compared to other low and middle income countries in the Global Network's Maternal Newborn Health Registry: an active, community-based, pregnancy surveillance mechanism. Reprod Health. 2015;12(Suppl 2):S15.

124. Patel A, Bucher S, Pusdekar Y, Esamai F, Krebs NF, Goudar SS, Chomba E, Garces A, Pasha O, Saleem S, et al. Rates and determinants of early initiation of breastfeeding and exclusive breast feeding at 42 days postnatal in six low and middle-income countries: A prospective cohort study. Reprod Health. 2015;12(Suppl 2):S10

125. Saleem S, Tikmani SS, McClure EM, Moore JL, Azam SI, Dhaded SM, Goudar SS, Garces A, Figueroa L, Marete I, et al. Trends and determinants of stillbirth in developing countries: results from the Global Network's Population-Based Birth Registry. Reprod Health. 2018;15(Suppl 1):100.

126. Short VL, Geller SE, Moore JL, McClure EM, Goudar SS, Dhaded SM, Kodkany BS, Saleem S, Naqvi F, Pasha O, et al. The Relationship between Body Mass Index in Pregnancy and Adverse Maternal, Perinatal, and Neonatal Outcomes in Rural India and Pakistan. Am J Perinatol. 2018;35(9):844-51.

127. Tikmani SS, Ali SA, Saleem S, Bann CM, Mwenechanya M, Carlo WA, Figueroa L, Garces AL, Krebs NF, Patel A, et al. Trends of antenatal care during pregnancy in low- and middle-income countries: Findings from the global network maternal and newborn health registry. Semin Perinatol. 2019:43(5):297-307.

128. Review of DHIS2 implementation experience: findings and lessons learnt. In. Bangkok: Thailand. Ministry of Health. 2016. https://accesstohealthfund.org/. Accesseed 10 May 2020.

129. Health Management Information Systems (HMIS): Review Survey on Data Availability in Electronic Systems for Maternal and Newborn Health Indicators in 24 USAID Priority Countries. In. Washington, DC: Unites States. Maternal and Child Survival Program. 2016. https://www.mcsprogram.org/. Accessed 25 May 2020.

130. Standard Operating Procedures (SOP) for Routine Registry OperationsImplementation, Establishment and Maintenance of Mother \& Child Health (MCH) Registry: Communication Strategies. In. Ramala: Palestina. National Institute of Public Health. 2017. https://www.pniph.org/. Accessed 20 May 2020.

131. DHIS2 District Health Information Software 2. https://www.dhis2.org/. Accessed 20 May 2020.

132. DHIS 2 Implementer guide: Applicable to master version. In. s.l.: DHIS2. 2020 https://docs.dhis2.org/. Accessed 20 May 2020

133. Begum T, Khan SM, Ferdous J, Parvez MM, Rahman A, Kumkum FA, Anwa I. Using DHIS 2 Software to Collect Health Data in Bangladesh. In. Chapel Hill: MEASURE Evaluation; 2019.

134. Rawlins B: Measurement Matters! Improving Routine RMNCAH Data for Better Outcomes. In. Washington, DC: Unites States. USAIDS. Maternal and Child Survival Program. 2019. https://www.mcsprogram.org/. Accessed 23 June 2020.

135. Singh GP: Improving Data for Decision-making: Leveraging Data Quality Audits in Haryana, India. In. Delhl: United States. Health Finance and Governance Project. 2014. https:/wnw.hfgproject.org/. Accessed 1 July 2020

136. Unicef: Health System Strengthening: Transforming the health information system in Bangladesh: Case Study Bangladesh. In. s.l.: UNICEF Regional Office for South Asia. 2019. https://www.unicef.org/. Accessed 2 July 2020.

137. Bergum B-I, Kusumasindra F, Øren M, Falch V, Sahraoui T: Analyzing DHIS2 as an information infrastructure. Information infrastructure. 2015. https:// www.uio.no/. Accessed 7 May 2020.

138. Valbø B: Introducing a complex health information system in a developing country: Case: The Gambia. Oslo: Unievrsity of Oslo. 2010. https://www.duo. uio.no/. Accessed 5 May 2020.

139. Manya A, Nielsen P. Reporting practices and data quality in health information systems in developing countries: an exploratory case study in Kenya. J Health Inform Dev Countries. 2016;10(1):114-26 28 ref 2016. 
140. Barron P, Peter J, LeFevre AE, Sebidi J, Bekker M, Allen R, Parsons AN, Benjamin P, Pillay Y. Mobile health messaging service and helpdesk for South African mothers (MomConnect): history, successes and challenges. BMJ Glob Health. 2018;3(Suppl 2):e000559.

141. Barron P, Pillay Y, Fernandes A, Sebidi J, Allen R. The MomConnect mHealth initiative in South Africa: Early impact on the supply side of $\mathrm{MCH}$ services. J Public Health Policy. 2016;37(Suppl 2):201-12.

142. Begum T, Khan SM, Adamou B, Ferdous J, Parvez MM, Islam MS, Kumkum FA, Rahman A, Anwar I. Perceptions and experiences with district health information system software to collect and utilize health data in Bangladesh: a qualitative exploratory study. BMC Health Serv Res. 2020;20(1): 465.

143. Beguy D, Elung'ata P, Mberu B, Oduor C, Wamukoya M, Nganyi B, Ezeh A. Health \& Demographic Surveillance System Profile: The Nairobi Urban Health and Demographic Surveillance System (NUHDSS). Int J Epidemiol. 2015;44(2):462-71.

144. Faujdar DS, Sahay S, Singh T, Jindal H, Kumar R. Public health information systems for primary health care in India: A situational analysis study. J Fam Med Prim Care. 2019;8(11):3640-6.

145. Hassan S, Vikanes A, Laine K, Zimmo K, Zimmo M, Bjertness E, Fosse E. Building a research registry for studying birth complications and outcomes in six Palestinian governmental hospitals. BMC Pregnancy Childbirth. 2017; 17(1):112.

146. Heekes A, Tiffin N, Dane P, Mutemaringa T, Smith M, Zinyakatira N, Barron P, Seebregts C, Boulle A. Self-enrolment antenatal health promotion data as an adjunct to maternal clinical information systems in the Western Cape Province of South Africa. BMJ Glob Health. 2018;3(Suppl 2):e000565.

147. LeFevre AE, Dane P, Copley CJ, Pienaar C, Parsons AN, Engelhard M, Woods D, Bekker M, Benjamin P, Pillay Y, et al. Unpacking the performance of a mobile health information messaging program for mothers (MomConnect) in South Africa: evidence on program reach and messaging exposure. BMJ Glob Health. 2018;3(Suppl 2):e000583.

148. Seebregts C, Dane P, Parsons AN, Fogwill T, Rogers D, Bekker M, Shaw V, Barron P. Designing for scale: optimising the health information system architecture for mobile maternal health messaging in South Africa (MomConnect). BMJ Glob Health. 2018;3(Suppl 2):e000563.

149. Xiong K, Kamunyori J, Sebidi J. The MomConnect helpdesk: how an interactive mobile messaging programme is used by mothers in South Africa. BMJ Glob Health. 2018;3(Suppl 2):e000578.

150. Venkateswaran M, Mørkrid K, Abu Khader K, Awwad T, Friberg IK, Ghanem B, Hijaz T, Frøen JF. Comparing individual-level clinical data from antenatal records with routine health information systems indicators for antenatal care in the West Bank: A cross-sectional study. Plos One. 2018;13(11): e0207813.

151. Seebregts C, Barron P, Tanna G, Benjamin P, Fogwill T. MomConnect: an exemplar implementation of the Health Normative Standards Framework in South Africa. South Afr Health Rev. 2016:2016:125-35 36 ref 2016.

152. OpenMRS: Open Medical Record System. 2020. https://openmrs.org/. Accessed 4 May 2020

153. Bashiri A, Ghazisaeedi M. Open MRS softwares: effective approaches in management of patients' health information. Int J Commun Med Publ Health. 2017:4(11):4.

154. Downey M, Mamlin B: OpenMRS Wiki. In. s.l.: OpenMRS. 2017. https://wiki. openmrs.org/. Accessed 4 may 2020.

155. Allen C, Jazayeri D, Miranda J, Biondich PG, Mamlin BW, Wolfe BA, Seebregts C, Lesh N, Tierney WM, Fraser HS. Experience in implementing the OpenMRS medical record system to support HIV treatment in Rwanda. Stud Health Technol Inform. 2007;129(Pt 1):382-6.

156. Mamlin BW, Biondich PG, Wolfe BA, Fraser H, Jazayeri D, Allen C, Miranda J, Tierney WM. Cooking up an open source EMR for developing countries: OpenMRS - a recipe for successful collaboration. AMIA Annu Symp Proc. 2006;2006:529-33.

157. Manders EJ, José E, Solis M, Burlison J, Nhampossa JL, Moon T. Implementing OpenMRS for patient monitoring in an HIV/AIDS care and treatment program in rural Mozambique. Stud Health Technol Inform. 2010; 160(Pt 1):411-5

158. Satti H, Motsamai S, Chetane P, Marumo L, Barry DJ, Riley J, McLaughlin MM, Seung KJ, Mukherjee JS. Comprehensive approach to improving maternal health and achieving MDG 5: report from the mountains of Lesotho. Plos One. 2012:7(8):e42700.
159. Thompson A, Castle E, Lubeck P, Makarfi PS. Experience implementing OpenMRS to support maternal and reproductive health in Northern Nigeria. Stud Health Technol Inform. 2010;160(Pt 1):332-6.

160. Tierney WM, Achieng M, Baker E, Bell A, Biondich P, Braitstein P, Kayiwa D, Kimaiyo S, Mamlin B, McKown B, et al. Experience implementing electronic health records in three East African countries. Stud Health Technol Inform. 2010;160(Pt 1):371-5.

161. Sistema Informático Perinatal. 2020. http://www.clap.ops-oms.org/. Accessed 15 May 2020

162. Fescina R: History of the Perinatal Information System. Making Pregnancy Safer. 2010. https://www.paho.org/. Accessed 15 May 2020.

163. Mainero L, Martínez G, Rubino M, De Mucio B, Díaz Rosello JL, Fescina R: Sistema informático perinatal (SIP): manual de uso del programa para el análisis y aprovechamiento de la información. Sci Publ; 1565-02 2010. Accessed 24 May 2020.

164. Bradley H, Tapia V, Kamb ML, Newman LM, Garcia PJ, Serruya SJ, Fort AL, Broutet N, Nelson R, Kirkcaldy RD, et al. Can the Perinatal Information System in Peru be used to measure the proportion of adverse birth outcomes attributable to maternal syphilis infection? Rev Panam Salud Publica. 2014;36(2):73-9.

165. Karolinski A, Mercer R, Bolzán A, Salgado P, Ocampo C, Nieto R, Birmingham M, Martínez G, Mainero L. Serruya S et al: [Foundations for the development and implementation of a women's and perinatal health information model for management in Latin AmericaFundamentos do desenvolvimento e implementação de um modelo de informação em saúde da materna e perinatal orientado à gestão na América Latina]. Rev Panam Salud Publica. 2018;42:e148.

166. Serruya SJ, Duran P, Martinez G, Romero M, Caffe S, Alonso M, Silveira MF. Maternal and congenital syphilis in selected Latin America and Caribbean countries: a multi-country analysis using data from the Perinatal Information System. Sex Health. 2015;12(2):164-9.

167. Levine R, Corbacio A, Konopka S, Saya U, Gilmartin C, Paradis J, Haas S. mHealth Compendium. 5. Arlington: United States Agency for International Development; 2015. http://www.africanstrategies4health.org/. Accessed 15 May 2020

168. Open Smart Register Platform (OpenSRP): Delivering a longitudinal patient record and decision support system for frontline health workers. 2020. https://www.who.int/reproductivehealth/topics/mhealth/openspr/en/. Accessed 27 May 2020.

169. THRIVE Indonesia: An Integrated, Mobile Health Information System Enhances Maternal And Neonatal Health Outcomes. 2020. https://sma rtcitiescouncil.com/resources/thrive-indonesia-integrated-mobile-healthinformation-system-enhances-maternal-and-neonatal-health. Accessed 20 May 2020.

170. OpenSRP: Open Smart Register Platform. 2020. http://smartregister.org/ index.html. Accessed 20 May 2020.

171. Kevin K, Inraini F, Ahmad Rafi J, Resty Asmauryanah A, Yusran L, Muhammad Abdi $H$, Benyamin $H$, Anuraj S. Midwife service coverage, quality of work, and client health improved after deployment of an OpenSRP-driven client management application in Indonesia. In: 5th International Conference on Health Sciences (ICHS 2018): 2019/11 2019: Atlantis Press; 2019:155-62. https://download.atlantis-press.com/proceedings/ichs-18/125921329. Accessed 20 May 2020.

172. Haddad SM, Souza RT, Cecatti JG. Mobile technology in health (mHealth) and antenatal care-Searching for apps and available solutions: A systematic review. Int J Med Inform. 2019;127:1-8.

173. Kalk E, Mehta U, Slogrove A, Jacob N, Myer L, Davies MA, Boulle A. Pregnancy exposure registry/birth defects surveillance programme in the Western Cape, South Africa: A model for low- and middle-income countries. Drug Saf. 2018:41(11):1212-3.

174. Mehta U, Heekes A, Kalk E, Boulle A. Assessing the value of Western Cape Provincial Government health administrative data and electronic pharmacy records in ascertaining medicine use during pregnancy. S Afr Med J. 2018; 108(5):439-43.

175. Mehta UC, van Schalkwyk C, Naidoo P, Ramkissoon A, Mhlongo O, Maharaj NR, Naidoo N, Fieggen K, Urban MF, Krog S, et al. Birth outcomes following antiretroviral exposure during pregnancy: Initial results from a pregnancy exposure registry in South Africa. South Afr J HIV Med. 2019;20(1):971.

176. Dheda M: Pregnancy Registers. In. Midrand: South Africa. National Department of Health. 2018. https://www.sahivsoc2018.co.za/. Accessed 20 May 2020. 
177. Epidemiology for Data Users (EDU) Trainer's Manual. In. Lusaka: Zambia. Ministry of Health. 2011. https://www.nastad.org/. Accessed 17 June 2020.

178. SmartCare. 2020. https://helpdesk.moh.gov.zm/. Accessed 15 june 2020.

179. Gumede-Moyo S, Todd J, Bond V, Mee P, Filteau S. A qualitative inquiry into implementing an electronic health record system (SmartCare) for prevention of mother-to-child transmission data in Zambia: a retrospective study. BMJ Open. 2019;9(9):e030428.

180. Mweebo K. Security of electronic health records in a resource limited setting: The case of smart-care electronic health record in Zambia. SRI Security Research Institute, Edith Cowan University. In: 3rd Australian eHealth Informatics and Security Conference. Perth, Australia; 2014. https://doi.org/10.4225/75/5798297631b47.

181. Footman K, Chersich M, Blaauw D, Campbell OM, Dhana A, Kavanagh J, Dumbaugh M, Thwala S, Bijlmakers L, Vargas E, et al. A systematic mapping of funders of maternal health intervention research 2000-2012. Global Health. 2014;10:72.

182. Fairlie L, Mehta UC: National pregnancy exposure registry for South Africa In. Edited by Buekens PM; 2020

183. Baiden R, Oduro A, Halidou T, Gyapong M, Sie A, Macete E, Abdulla S, Owusu-Agyei S, Mulokozi A, Adjei A, et al. Prospective observational study to evaluate the clinical safety of the fixed-dose artemisinin-based combination Eurartesim ${ }^{\oplus}$ (dihydroartemisinin/piperaquine), in public health facilities in Burkina Faso, Mozambique, Ghana, and Tanzania. Malar J. 2015; 14:160.

184. Bonhoeffer J, Kochhar S, Hirschfeld S, Heath PT, Jones CE, Bauwens J, Honrado Á, Heininger U, Muñoz FM, Eckert L, et al. Global alignment of immunization safety assessment in pregnancy - The GAIA project. Vaccine. 2016;34(49):5993-7.

185. Frøen JF, Myhre SL, Frost MJ, Chou D, Mehl G, Say L, Cheng S, Fjeldheim I, Friberg IK, French $S$, et al. eRegistries: Electronic registries for maternal and child health. BMC Pregnancy Childbirth. 2016;16:11.

186. Crawford M, van Wyk J, Aboud M, Vannappagari V, Romach B, Curtis L, Wynne B, de Ruiter A, Smith K, Payvandi N. Postmarketing Surveillance of Pregnancy Outcomes With Dolutegravir Use. J Acquir Immune Defic Syndr. 2020;83(1):e2-5

187. IMPAACT Studies. 2020. https://impaactnetwork.org/studies/index.asp. Accessed 26 May 2020

188. Tricco AC, Lillie E, Zarin W, O'Brien K, Colquhoun H, Kastner M, Levac D, Ng C, Sharpe JP, Wilson K, et al. A scoping review on the conduct and reporting of scoping reviews. BMC Med Res Methodol. 2016;16:15.

189. Sobanjo-Ter Meulen A, Liljestrand J, Lawn JE, Hombach J, Smith J, Dickson KE, Munoz FM, Omer SB, Williams BA, Klugman KP. Preparing to introduce new maternal immunizations in low- and lower-middle-income countries: A report from the Bill \& Melinda Gates Foundation convening "Allies in Maternal and Newborn Care"; May 3-4, 2018. Vaccine. 2020;38(28):4355-61.

\section{Publisher's Note}

Springer Nature remains neutral with regard to jurisdictional claims in published maps and institutional affiliations.

Ready to submit your research? Choose BMC and benefit from:
- fast, convenient online submission
- thorough peer review by experienced researchers in your field
- rapid publication on acceptance
- support for research data, including large and complex data types
- gold Open Access which fosters wider collaboration and increased citations
- maximum visibility for your research: over 100M website views per year
At BMC, research is always in progress.
Learn more biomedcentral.com/submissions

